\title{
Estimation of Reliability of Stress-strength for a Kumaraswamy Distribution based on Progressively Censored Sample
}

\author{
Akram Kohansal* and Ramin Kazemi \\ Imam Khomeini International University \\ Received: 5/14/2020 Approved: 10/13/2020
}

\begin{abstract}
The estimation of $R=P(X<Y)$ in the case that $X$ and $Y$ are two independent Kumaraswamy distributed random variables with different parameters for progressively Type-II censored samples is studied. First assuming the same second shape parameters of two distributions, the maximum likelihood estimation and different confidence intervals are considered. Moreover, in case the second shape parameters of two distributions are known, MLE, UMVUE, Bayes estimation of $R$ and confidence intervals are derived. Finally, the Maximum Likelihood and Bayes estimations of $R$ in general case are obtained. Performance comparisons of different methods are carried out utilizing Monte Carlo simulations. Besides, the proposed approach is employed for reliability analysis on a real strength-stress dataset to demonstrate its application.
\end{abstract}

Keywords. Kumaraswamy distribution; Progressive Type-II censoring; Bayesian estimator; Confidence interval; Monte Carlo simulation; Maximum likelihood estimator.

MSC 2010: 62F10, 62F15, 62N02.

${ }^{*}$ Corresponding author 


\section{Introduction}

Parameter estimation of $R=P(X<Y)$ where the stress $X$ and the strength $Y$ are both random variables is a problem of great interest in reliability analysis. A failure occurs in an arbitrary system whenever the system strength is lower than the stress applied to it. In the stress-strength modeling, study of $R$ is of importance for researchers to analyze data in different fields of study such as engineering, biology, economics and etc. In mechanical reliability of a system, $R$ could be a measure of system tolerance if $Y$ is the component strength which is subject to a stress $X$. For another example, consider $X$ as the gas pressure inside a chamber generated by ignition of a solid propellant and $Y$ as the strength of the chamber. Then $R$ represents the probability of successful firing of the engine. In these two cases, $R$ is indeed a measure of system performance. It should be noted that in addition to reliability analysis estimation of $R$ is of broad applications in lots of research areas as it provides a general measure of the difference between two populations. For example, in medical diagnostics the probability $P(X<Y)$ is also known as the area under the receiver operating characteristic curve, Li and Fine (2010), Li and Ma (2011).

Considerable researches have been devoted to estimate the strength stress parameter in statistics, starting with the pioneering work of Birnbaum (1956). In Birnbaum (1956), the author could find a link between the traditional Mann-Whitney statistic and $R$ parameter. From that day forward, the estimation of $R$ has been studied considering different distributions for stress and strength parameters. Kotz, et al. (2003) presented the best pioneering review monograph to develop such this reliability model. Some recent works on the stress-strength model have been carried out by Raqab and Kundu (2005), Asgharzadeh, et al. (2011), Shoaee and Khorram (2015), Mirjalili, et al. (2016), Ahmadi and Ghafouri (2019). In spite of extensive works to develop the stress-strength models under complete samples (Nadar, et al. (2014), Nadar and Kizilaslan (2014), Wang, et al. (2016)), less efforts have been made to the case of censored data (Asgharzadeh, et al. (2011), Shoaee and Khorram (2015), Mirjalili, et al. (2016), Ahmadi and Ghafouri (2019)). However, in many practical situations, it would be possible that rather than complete samples just censored samples from both populations are observed. An example was discussed by Surles and Padgett $(1998,2001)$, Kundu and Gupta (2006) and Raqab and Kundu (2005). In this example, the strengths of carbon fibers at different gauge lengths were compared by 
computing $P(X<Y)$, where $X$ and $Y$ denote the strengths of the fibre at two different gauge lengths.

Type-I and Type-II censoring schemes are two conventional censoring methods. In Type-I censoring, the test is finished after passing a predetermined time on the test and in Type-II censoring, the test is ended after observing a pre-chosen number of failures. Unfortunately removal of active units is prohibited during the test in both of the aforementioned censoring schemes. In the progressive censoring method, removing active units during the experiment is allowed. Among various censoring schemes in the last decade, the widely used one is the progressive Type-II censoring that is a combination of the Type-II and progressive censoring schemes. It can be described as follows: Suppose $N$ units are placed on a life test and the experimenter would decide beforehand the quantity $n$, the number of failures to be observed. At the first failure time, $R_{1}$ units of $N-1$ surviving units are randomly removed from the experiment. Similarly when the second failure occurs, from the remaining $N-R_{1}-1$ units a number of $R_{2}$ units are randomly selected and removed. Finally, at the $n$-th failure, all the remaining surviving units $R_{n}=N-n-R_{1}-\ldots-R_{n-1}$ are removed from the experiment. Consequently, a progressive Type-II censoring method consists of $n$, and $\left(R_{1}, \ldots, R_{n}\right)$, such that $R_{1}+\ldots+R_{n}=N-n$. It is clear that this method includes the conventional Type-II right censoring scheme (when $R_{1}=\ldots=R_{n-1}=0$ and $R_{n}=N-n$ ) and complete sampling scheme (when $N=n$ and $R_{1}=\ldots=R_{n}=0$ ). Further details on the progressively censoring method and the corresponding references are brought in a book written by Balakrishnan and Aggarwala (2000).

Utilizing progressively Type-II censoring method, this paper studies the inference of $R=P(X<Y)$ when $X$ and $Y$ come from two independent Kumaraswamy distributions (KuD) with different parameters. The probability density function, cumulative density function and failure rate function of a $\mathrm{KuD}$ with the first and second shape parameters $\alpha$ and $\lambda$, are respectively defined by:

$$
\begin{array}{ll}
f(x)=\alpha \lambda x^{\lambda-1}\left(1-x^{\lambda}\right)^{\alpha-1}, & 0<x<1, \quad \alpha, \lambda>0 \\
F(x)=1-\left(1-x^{\lambda}\right)^{\alpha}, & 0<x<1, \quad \alpha, \lambda>0 \\
H(x)=\frac{\alpha \lambda x^{\lambda-1}}{1-x^{\lambda}}, & 0<x<1, \quad \alpha, \lambda>0
\end{array}
$$

Herein we refer to a $\mathrm{KuD}$ with the pdf $(1)$ as $K u(\alpha, \lambda)$. Figure 1 depicts 

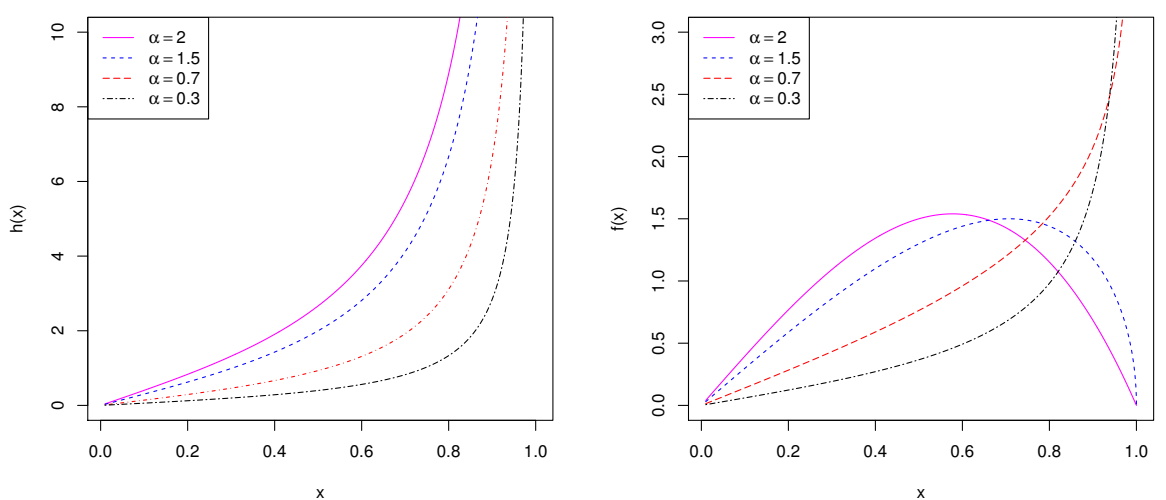

Figure 1. Shape of density function (right) and failure rate function (left) of KuD when $\lambda=2$.

the density function and the failure rate function of $\mathrm{KuD}$. As shown in this figure, $\mathrm{KuD}$ has an increasing failure rate function. Hence, if an empirical study on the underlying distribution of a data set shows that the failure rate function is increasing, the $\mathrm{KuD}$ could be utilized for analysis of such a data set. The outcomes of many natural phenomena that have lower and upper bounds could be described by the Kumaraswamy distribution, for example atmospheric temperatures, scores obtained on a test, heights of individuals, economic data (such as unemployment data), and etc.

Jones investigated some basic properties of this distribution Jones (2009). Lemonte proposed an improved point estimation for this distribution Lemonte (2011) while Mitnik presented some of its interesting newer properties Mitnik (2013). Recently, Nadar, et al. (2013) accomplished a statistical analysis from the Kumaraswamy distribution based on record values. Later, Nadar, et al. (2014) studied estimation of $R=P(Y<X)$ in case that two independent random variables $X$ and $Y$ are from Kumaraswamy distributions with different shape parameters. In some experiments, such as meteorology, hydrology, sports and life-tests, it is plausible to make measurements sequentially of which those measured values being smaller (or larger) than all previous ones are kept. Such a measured data is known as "Record Data". Hence, the final number of available measurements is noticeably smaller than the complete sample size. This "measurement saving" would be of importance as the measurements of the experiments are costly in case the entire 
sample was destroyed. Let $X_{1}, X_{2}, \ldots$ be a sequence of i.i.d. random variables. An upper record value is an observed sample $X_{j}$ whose value exceeds the values of all previous observations. Assuming $X$ and $Y$ are two independent Kumaraswamy random variables with different shape parameters, and having the first $n$ and $m$ upper record values observed from the $X$ and $Y$, Nadar and Kizilaslan (2014) obtain the estimation of $P(X<Y)$ using the maximum likelihood and Bayesian approaches. More recently, Wang, et al. (2016) explained many statistical inference of a random variable which follows a Kumaraswamy distribution. This paper infers different estimations based on complete sample not censored sample.

In the current study, we consider the inference of $R=P(X<Y)$ when progressively Type-II censored samples are observed from Kumaraswamy distributed random variables $X$ and $Y$. The problem is formulated as follows: let $X$ and $Y$ be independent Kumaraswamy random variables with different parameters. Two progressive censoring schemes for $X$ and $Y$ are $\left\{N, n, R_{1}, \ldots, R_{n}\right\}$ and $\left\{M, m, S_{1}, \ldots, S_{m}\right\}$, respectively. Then, $\left\{X_{1: n: N}, \ldots, X_{n: n: N}\right\}$ and $\left\{Y_{1: m: M}, \ldots, Y_{m: m: M}\right\}$ are the progressively censored samples from $X$ and $Y$, respectively. Now, the problem to be solved is estimation of $R=P(X<$ $Y)=\frac{\alpha}{\alpha+\beta}$ when the mentioned progressive censored samples are observed.

The layout of this paper is as follows. Assuming the common second shape parameter, some approaches to estimate $R$ are proposed in Section 2 where the ML estimation of $R$, asymptotic and two bootstrap confidence intervals, Bayes estimation and the associated credible interval are provided. Estimation of $R$ in case of the known common second shape parameters is discussed in Section 3. In this section MLE, uniformly minimum variance unbiased estimator (UMVUE), the exact confidence interval, asymptotic and two bootstrap confidence intervals, Bayes estimation and the associated credible interval of $R$ are discussed. The general case of estimating $R$ is studied in Section 4 where the ML and Bayes estimations of $R$ are proposed. Simulation results based on Monte Carlo experiments and real data analysis are reported in Section 5, and conclusion is presented in Section 6.

\section{Estimation of $\mathbf{R}$ with Common Second Shape Parameter}

Assuming the common second shape parameter $\lambda$, for $X$ and $Y$, we investigate the properties of $R$ in this section. The general case will be discussed 
in Section 4.

\subsection{Maximum Likelihood Estimation of $\mathbf{R}$}

Let $X \sim K u(\alpha, \lambda)$ and $Y \sim K u(\beta, \lambda)$ be independent random variables. It is straightforward to show that the reliability function is $R=P(X<Y)=$ $\frac{\alpha}{\alpha+\beta}$. Estimation of $R$ in case of the progressive Type-II censored samples is our purpose.

First, we have to obtain the MLEs of $\alpha, \beta$ and $\lambda$ to derive the MLE of $R$. Let $\left\{X_{1: n: N}, \ldots, X_{n: n: N}\right\}$ and $\left\{Y_{1: m: M}, \ldots, Y_{m: m: M}\right\}$ be a progressively censored sample from $K u(\alpha, \lambda)$ and $K u(\beta, \lambda)$ under the progressive censoring scheme $\left\{N, n, R_{1}, R_{2}, \ldots, R_{n}\right\}$ and $\left\{Y_{1: m: M}, \ldots, Y_{m: m: M}\right\}$, respectively. Hence, the likelihood function of $\alpha, \beta$ and $\lambda$ is defined as

$$
L(\alpha, \beta, \lambda)=\left[c_{1} \prod_{i=1}^{n} f\left(x_{i}\right)\left[1-F\left(x_{i}\right)\right]^{R_{i}}\right] \times\left[c_{2} \prod_{j=1}^{m} f\left(y_{j}\right)\left[1-F\left(y_{j}\right)\right]^{S_{j}}\right],
$$

where

$$
\begin{aligned}
& c_{1}=N\left(N-R_{1}-1\right) \ldots\left(N-R_{1}-\ldots-R_{n-1}-n+1\right), \\
& c_{2}=M\left(M-S_{1}-1\right) \ldots\left(M-S_{1}-\ldots-S_{m-1}-m+1\right) .
\end{aligned}
$$

The likelihood function of the observed data is:

$$
\begin{aligned}
L(\text { data } \mid \alpha, \beta, \lambda)= & c_{1} c_{2} \alpha^{n} \beta^{m} \lambda^{n+m}\left(\prod_{i=1}^{n} x_{i}^{\lambda-1}\right)\left(\prod_{j=1}^{m} y_{j}^{\lambda-1}\right) \\
& \times\left(\prod_{i=1}^{n}\left(1-x_{i}^{\lambda}\right)^{\alpha\left(R_{i}+1\right)-1}\right)\left(\prod_{j=1}^{m}\left(1-y_{j}^{\lambda}\right)^{\beta\left(S_{j}+1\right)-1}\right) .
\end{aligned}
$$

Therefore, the log-likelihood function is as follows: 


$$
\begin{aligned}
\ell(\alpha, \beta, \lambda) & =n \log (\alpha)+m \log (\beta)+(n+m) \log (\lambda)+(\lambda-1) \\
& \times\left(\sum_{i=1}^{n} \log \left(x_{i}\right)+\sum_{j=1}^{m} \log \left(y_{j}\right)\right)+\sum_{i=1}^{n}\left(\alpha\left(R_{i}+1\right)-1\right) \log \left(1-x_{i}^{\lambda}\right) \\
& +\sum_{j=1}^{m}\left(\beta\left(S_{j}+1\right)-1\right) \log \left(1-y_{j}^{\lambda}\right)+\text { Constant. }
\end{aligned}
$$

The maximum likelihood estimations of $\alpha, \beta$ and $\lambda$, which are respectively denoted by $\widehat{\alpha}, \widehat{\beta}$ and $\widehat{\lambda}$, are achieved by solving the equation below

$$
\begin{aligned}
\frac{\partial \ell}{\partial \alpha} & =\frac{n}{\alpha}+\sum_{i=1}^{n}\left(R_{i}+1\right) \log \left(1-x_{i}^{\lambda}\right)=0 \\
\frac{\partial \ell}{\partial \beta} & =\frac{m}{\beta}+\sum_{j=1}^{m}\left(S_{j}+1\right) \log \left(1-y_{j}^{\lambda}\right)=0 \\
\frac{\partial \ell}{\partial \lambda}= & \frac{n+m}{\lambda}+\sum_{i=1}^{n} \log \left(x_{i}\right)+\sum_{j=1}^{m} \log \left(y_{j}\right)-\sum_{i=1}^{n} \frac{\left(\alpha\left(R_{i}+1\right)-1\right) x_{i}^{\lambda} \log \left(x_{i}\right)}{1-x_{i}^{\lambda}} \\
& -\sum_{j=1}^{m} \frac{\left(\beta\left(S_{j}+1\right)-1\right) y_{j}^{\lambda} \log \left(y_{j}\right)}{1-y_{j}^{\lambda}}=0 .
\end{aligned}
$$

From (2) and (3), we obtain

$$
\widehat{\alpha}(\lambda)=-\frac{n}{\sum_{i=1}^{n}\left(R_{i}+1\right) \log \left(1-x_{i}^{\lambda}\right)}, \quad \widehat{\beta}(\lambda)=-\frac{m}{\sum_{j=1}^{m}\left(S_{j}+1\right) \log \left(1-y_{j}^{\lambda}\right)},
$$

and $\hat{\lambda}$ can be found as the solution of the non-linear equation $k(\lambda)=\lambda$, where 


$$
\begin{aligned}
k(\lambda)=(n+m)\left\{\sum_{i=1}^{n} \log \left(x_{i}\right)\right. & \left(-1+\frac{\left(\alpha\left(R_{i}+1\right)-1\right) x_{i}^{\lambda}}{1-x_{i}^{\lambda}}\right) \\
& \left.+\sum_{j=1}^{m} \log \left(y_{j}\right)\left(-1+\frac{\left(\beta\left(S_{j}+1\right)-1\right) y_{j}^{\lambda}}{1-y_{j}^{\lambda}}\right)\right\}^{-1} .
\end{aligned}
$$

Because, $\hat{\lambda}$ is a fixed point solution of the above non-linear equation, it can be iteratively obtained as $k\left(\lambda_{(j)}\right)=\lambda_{(j+1)}$, where $\lambda_{(j)}$ is the value of $\hat{\lambda}$ in the $j$-th iteration. This iterative procedure is finished if $\left|\lambda_{(j)}-\lambda_{(j+1)}\right|$ gets small enough. Now, $\widehat{\alpha}$ and $\widehat{\beta}$ can be calculated after $\widehat{\lambda}$ is obtained. Finally, the maximum likelihood estimation of $R$ would be

$$
\widehat{R}=\frac{\widehat{\alpha}}{\widehat{\alpha}+\widehat{\beta}}
$$

\subsection{Asymptotic Confidence Interval}

Here, we present some approaches to obtain the asymptotic distributions of both $\widehat{\theta}=(\widehat{\alpha}, \widehat{\beta}, \widehat{\lambda})$ and $\widehat{R}$. We denote the expected Fisher information matrix of $\theta=(\alpha, \beta, \lambda)$ as $J(\theta)=-E(I(\theta))$, where $I=\left[I_{i j}\right], i, j=1,2,3$ is the observed information matrix. According to the table of the integrals from Gradshteyn and Ryzhik (1994) (formulae 4.253(1) and 4.261(21)), we have

$$
\begin{aligned}
& J_{11}=\frac{n}{\alpha^{2}}, \quad J_{22}=\frac{m}{\beta^{2}}, \quad J_{12}=J_{21}=0, \\
& J_{13}=J_{31}=\frac{\alpha}{\lambda} \sum_{i=1}^{n}\left(R_{i}+1\right) C_{i-1} \\
& \quad \times \sum_{k=1}^{i} a_{i, k} B\left(2, \alpha \eta_{k}-1\right)\left[\psi(2)-\psi\left(\alpha \eta_{k}+1\right)\right], \quad \text { for } \alpha \eta_{k}>1
\end{aligned}
$$




$$
\begin{aligned}
J_{23} & =J_{32}=\frac{\beta}{\lambda} \sum_{j=1}^{m}\left(S_{j}+1\right) C_{j-1}^{\prime} \\
& \times \sum_{k=1}^{j} a_{j, k}^{\prime} B\left(2, \beta \eta_{k}^{\prime}-1\right)\left[\psi(2)-\psi\left(\beta \eta_{k}^{\prime}+1\right)\right], \quad \text { for } \beta \eta_{k}^{\prime}>1 \\
J_{33} & =\frac{n+m}{\lambda^{2}}+\frac{\alpha}{\lambda^{2}} \sum_{i=1}^{n}\left(\alpha\left(R_{i}+1\right)-1\right) C_{i-1} \sum_{k=1}^{i} a_{i, k} B\left(2, \alpha \eta_{k}-2\right) \\
& \times\left\{\psi^{\prime}(2)-\psi^{\prime}\left(\alpha \eta_{k}\right)+\left[\psi(2)-\psi\left(\alpha \eta_{k}\right)\right]^{2}\right\} \\
& +\frac{\beta}{\lambda^{2}} \sum_{j=1}^{m}\left(\beta\left(S_{j}+1\right)-1\right) C_{j-1}^{\prime} \sum_{k=1}^{j} a_{j, k}^{\prime} B\left(2, \beta \eta_{k}^{\prime}-2\right) \\
& \times\left\{\psi^{\prime}(2)-\psi^{\prime}\left(\beta \eta_{k}^{\prime}\right)+\left[\psi(2)-\psi\left(\beta \eta_{k}^{\prime}\right)\right]^{2}\right\} \quad \text { for } \alpha \eta_{k}, \beta \eta_{k}^{\prime}>2,
\end{aligned}
$$

where $\psi(x)=\frac{d}{d x} \Gamma(x), \psi^{\prime}(x)=\frac{d^{2}}{d x^{2}} \Gamma(x), B(x, y)=\frac{\Gamma(x) \Gamma(y)}{\Gamma(x+y)}, \eta_{d}=n-d+$ $1-\sum_{l=d}^{n} R_{l}, \eta_{d}^{\prime}=m-d+1-\sum_{l=d}^{m} S_{l}, C_{i-1}=\prod_{d=1}^{i} \eta_{d}, C_{j-1}^{\prime}=\prod_{d=1}^{j} \eta_{d}^{\prime}$, $a_{i, d}=\prod_{l=1, l \neq i}^{d} \frac{1}{\eta_{l}-\eta_{i}}, a_{j, d}^{\prime}=\prod_{l=1, l \neq j}^{d} \frac{1}{\eta_{l}^{\prime}-\eta_{i}^{\prime}}$ (for more details see Balakrishnan and Aggarwala (2000)).

Theorem 1. As $n \rightarrow \infty$ and $m \rightarrow \infty$ then

$$
[\sqrt{n}(\widehat{\alpha}-\alpha) \sqrt{m}(\widehat{\beta}-\beta) \sqrt{n}(\widehat{\lambda}-\lambda)]^{T} \stackrel{D}{\longrightarrow} N_{3}\left(0, \mathbf{A}^{-1}(\alpha, \beta, \lambda)\right),
$$

where $\mathbf{A}(\alpha, \beta, \lambda)$ and $\mathbf{A}^{-1}(\alpha, \beta, \lambda)$ are symmetric matrices and

$$
\begin{gathered}
\mathbf{A}(\alpha, \beta, \lambda)=\left(\begin{array}{ccc}
a_{11} & 0 & a_{13} \\
& a_{22} & a_{23} \\
& & a_{33}
\end{array}\right), \\
\mathbf{A}^{-1}(\alpha, \beta, \lambda)=\frac{1}{|\mathbf{A}(\alpha, \beta, \lambda)|}\left(\begin{array}{ccc}
b_{11} & b_{12} & b_{13} \\
& b_{22} & b_{23} \\
& & b_{33}
\end{array}\right),
\end{gathered}
$$


in which

$$
\begin{gathered}
a_{11}=\lim _{n, m \rightarrow \infty} \frac{J_{11}}{n}, \quad a_{13}=\lim _{n, m \rightarrow \infty} \frac{J_{13}}{n}, \quad a_{22}=\lim _{n, m \rightarrow \infty} \frac{J_{22}}{m}, \\
a_{23}=\lim _{n, m \rightarrow \infty} \frac{J_{23}}{\sqrt{n m}}, \quad a_{33}=\lim _{n, m \rightarrow \infty} \frac{J_{33}}{n}, \\
\text { and }|\mathbf{A}(\alpha, \beta, \lambda)|=a_{11} a_{22} a_{33}-a_{11} a_{23}^{2}-a_{13}^{2} a_{22}, \\
b_{11}=a_{22} a_{33}-a_{23}^{2}, \quad b_{12}=a_{13} a_{23}, \quad b_{13}=-a_{13} a_{22}, \\
b_{22}=a_{11} a_{33}-a_{13}^{2}, \quad b_{23}=-a_{11} a_{23}, \quad b_{33}=a_{11} a_{22} .
\end{gathered}
$$

Proof. Following the asymptotic normality of the MLE, the theorem is proved.

Theorem 2. As $n, m \rightarrow \infty$, and $n / m \rightarrow p$ then

$$
\sqrt{n}(\widehat{R}-R) \stackrel{D}{\longrightarrow} N(0, B)
$$

where

$$
B=\frac{\beta^{2} b_{11}+\alpha^{2} p b_{22}-2 \alpha \beta \sqrt{p} b_{12}}{|\mathbf{A}(\alpha, \beta, \lambda)|(\alpha+\beta)^{4}} .
$$

Proof. We can easily show that $E(\sqrt{n}(\widehat{R}-R)) \rightarrow 0$ as $n, m \rightarrow \infty$, and $n / m \rightarrow p$ and that

$$
\begin{aligned}
\operatorname{Var}(\sqrt{n}(\widehat{R}-R)) & =E(\sqrt{n}(\widehat{R}-R))^{2} \\
= & E\left\{\frac { 1 } { ( \widehat { \alpha } + \widehat { \beta } ) ^ { 2 } ( \alpha + \beta ) ^ { 2 } } \left[\beta^{2}[\sqrt{n}(\widehat{\alpha}-\alpha)]^{2}+\alpha^{2}(n / m)[\sqrt{m}(\widehat{\beta}-\beta)]^{2}\right.\right. \\
& -2 \alpha \beta \sqrt{n / m}[\sqrt{n}(\widehat{\alpha}-\alpha) \sqrt{m}(\widehat{\beta}-\beta)]]\} .
\end{aligned}
$$

As $n, m \rightarrow \infty$, and $n / m \rightarrow p$,

$$
\operatorname{Var}(\sqrt{n}(\widehat{R}-R)) \rightarrow B=\frac{\beta^{2} b_{11}+\alpha^{2} p b_{22}-2 \alpha \beta \sqrt{p} b_{12}}{|\mathbf{A}(\alpha, \beta, \lambda)|(\alpha+\beta)^{4}},
$$

employing Theorem 2, the consistency and asymptotic normality of MLE are obtained. 
From Theorem 2, the asymptotic confidence interval of $R$ is constructed. Moreover, utilizing the maximum likelihood estimations of $\alpha, \beta$ and $\lambda, B$ is estimated. Hence, a $100(1-\gamma) \%$ asymptotic confidence interval for $R$ is as follows:

$$
\left(\widehat{R}-z_{1-\frac{\gamma}{2}} \frac{\sqrt{\widehat{B}}}{\sqrt{n}}, \widehat{R}+z_{1-\frac{\gamma}{2}} \frac{\sqrt{\widehat{B}}}{\sqrt{n}}\right),
$$

where $z_{\gamma}$ is $100 \gamma$-th percentile of $N(0,1)$.

\subsection{Confidence Interval Based on Bootstrap Procedures}

As the sampling distribution of $\widehat{R}$ is not available in case $\lambda$ is unknown, the bootstrapping approach can be an alternative method instead of the one described in the Section 2.2 to develop an approximated confidence interval of the parameter $R$. Furthermore, it is obvious that in case of small sample sizes the performance of asymptotic confidence intervals gets degraded. Hence, based on the non-parametric bootstrap method two confidence intervals are proposed: (i) bootstrap-p method, which is referred to as Boot-p method and is originated from the basic idea of Efron (1982) and (ii) bootstrap-t method, which is referred to as Boot-t method, and is originated from the idea of Hall (1988).

\section{(i) Boot-p Method}

1. Generate a bootstrap sample of size $n,\left\{x_{1}^{*}, \ldots, x_{n}^{*}\right\}$ from $\left\{x_{1}, \ldots, x_{n}\right\}$ and generate a bootstrap sample of size $m,\left\{y_{1}^{*}, \ldots, y_{m}^{*}\right\}$ from $\left\{y_{1}, \ldots, y_{m}\right\}$. Based on $\left\{x_{1}^{*}, \ldots, x_{n}^{*}\right\}$ and $\left\{y_{1}^{*}, \ldots, y_{m}^{*}\right\}$, calculate the bootstrap estimation of $R$ that is $\widehat{R}^{*}$, utilizing (4).

2. Iterate 1 NBOOT times.

3. Let $G^{*}(x)=P\left(\widehat{R}^{*} \leq x\right)$ be the cumulative distribution function of $\widehat{R}^{*}$. Define $\widehat{R}_{B p}(x)=G^{*-1}(x)$ for a given $x$. The $100(1-\gamma) \%$ confidence interval of $R$ is approximately obtained by:

$$
\left(\widehat{R}_{B p}\left(\frac{\gamma}{2}\right), \widehat{R}_{B p}\left(1-\frac{\gamma}{2}\right)\right)
$$

\section{(ii) Boot-t Method}

1. From the samples $\left\{x_{1}, \ldots, x_{n}\right\}$ and $\left\{y_{1}, \ldots, y_{m}\right\}$, compute $\widehat{R}$. 
2. Same as in Boot-p method, first generate bootstrap sample $\left\{x_{1}^{*}, \ldots, x_{n}^{*}\right\}$ and $\left\{y_{1}^{*}, \ldots, y_{m}^{*}\right\}$ and then calculate $\widehat{R}^{*}$, the bootstrap estimation of $R$. Also, compute the statistic:

$$
T^{*}=\frac{\sqrt{n}\left(\widehat{R}^{*}-\widehat{R}\right)}{\sqrt{V\left(\widehat{R}^{*}\right)}} .
$$

Compute $V\left(\widehat{R}^{*}\right)$ using Theorem 2 .

3. Iterate 1 and 2 NBOOT times.

4. Let $H(x)=P\left(T^{*} \leq x\right)$ be the cumulative distribution function of $T^{*}$. For a given $x$, let

$$
\widehat{R}_{B t}(x)=\widehat{R}+n^{-\frac{1}{2}} H^{-1}(x) \sqrt{V(\widehat{R})} .
$$

The $100(1-\gamma) \%$ confidence interval of $R$ is approximately obtained by:

$$
\left(\widehat{R}_{B t}\left(\frac{\gamma}{2}\right), \widehat{R}_{B t}\left(1-\frac{\gamma}{2}\right)\right)
$$

\subsection{Bayes Estimation of $R$}

Assuming the parameters $\alpha, \beta$ and $\lambda$ are random variables, in this section we develop the Bayesian inference of $R$. We mainly discuss Bayes estimations and the associated credible intervals of $R$. Also, let suppose the priors of $\alpha$, $\beta$ and $\lambda$ are independent gamma distributions as follows:

$$
\begin{array}{lll}
\pi_{1}(\alpha) \propto \alpha^{a_{1}-1} e^{-b_{1} \alpha}, & & \alpha>0, a_{1}>0, b_{1}>0, \\
\pi_{2}(\beta) \propto \beta^{a_{2}-1} e^{-b_{2} \beta}, & & \beta>0, a_{2}>0, b_{2}>0, \\
\pi_{3}(\lambda) \propto \lambda^{a_{3}-1} e^{-b_{3} \lambda}, & & \lambda>0, a_{3}>0, b_{3}>0 .
\end{array}
$$

Given the observed sample, the joint posterior density function of $\alpha, \beta$ and $\lambda$ is

$$
\pi(\alpha, \beta, \lambda \mid \text { data })=\frac{L(\text { data } \mid \alpha, \beta, \lambda) \pi_{1}(\alpha) \pi_{2}(\beta) \pi_{3}(\lambda)}{\int_{0}^{\infty} \int_{0}^{\infty} \int_{0}^{\infty} L(\text { data } \mid \alpha, \beta, \lambda) \pi_{1}(\alpha) \pi_{2}(\beta) \pi_{3}(\lambda) d \alpha d \beta d \lambda}
$$

From (5), it is obvious that the Bayes estimate will not be analytically obtained. Consequently, we utilize the Gibbs and Metropolis sampling methods 
to calculate the Bayes estimation and the associated credible interval of $R$. The posterior pdfs of $\alpha$ and $\beta$ can be obtained as:

$$
\begin{aligned}
& \alpha \mid \beta, \lambda, \text { data } \sim \Gamma\left(n+a_{1}, b_{1}+\sum_{i=1}^{n}\left(R_{i}+1\right) \log \left(\frac{1}{1-x_{i}^{\lambda}}\right)\right), \\
& \beta \mid \alpha, \lambda, \text { data } \sim \Gamma\left(m+a_{2}, b_{2}+\sum_{j=1}^{m}\left(S_{j}+1\right) \log \left(\frac{1}{1-y_{j}^{\lambda}}\right)\right),
\end{aligned}
$$

and

$$
\pi(\lambda \mid \alpha, \beta, \text { data }) \propto \lambda^{n+m+a_{3}-1} e^{-b_{3} \lambda}\left(\prod_{i=1}^{n} \frac{x_{i}^{\lambda-1}}{1-x_{i}^{\lambda}}\right)\left(\prod_{j=1}^{m} \frac{y_{j}^{\lambda-1}}{1-y_{j}^{\lambda}}\right) .
$$

Because $\lambda$ has an unknown posterior pdf, to generate random numbers from the posterior pdf of $\lambda$, the Metropolis-Hastings method is employed. Therefore, the Gibbs sampling's algorithm is as follows:

1. Start with an initial guess $\left(\alpha_{(0)}, \beta_{(0)}, \lambda_{(0)}\right)$.

2. Set $t=1$.

3. Generate $\lambda_{(t)}$ from $\pi\left(\lambda \mid \alpha_{(t-1)}, \beta_{(t-1)}\right.$, data).

4. Generate $\alpha_{(t)}$ from $\Gamma\left(n+a_{1}, b_{1}+\sum_{i=1}^{n}\left(R_{i}+1\right) \log \left(\frac{1}{1-x_{i}^{\lambda}(t-1)}\right)\right)$.

5. Generate $\beta_{(t)}$ from $\Gamma\left(m+a_{2}, b_{2}+\sum_{j=1}^{m}\left(S_{j}+1\right) \log \left(\frac{1}{1-y_{j}^{\lambda}(t-1)}\right)\right)$.

6. Compute $R_{(t)}=\frac{\alpha_{(t)}}{\alpha_{(t)}+\beta_{(t)}}$.

7. Set $t=t+1$.

8. Iterate $3-7, T$ times.

As a result, the mean and variance of the posterior pdf of $R$ are approximately given by:

$\widehat{E}(R \mid$ data $)=\frac{1}{T} \sum_{t=1}^{T} R_{(t)}, \widehat{\operatorname{Var}}(R \mid$ data $)=\frac{1}{T} \sum_{t=1}^{T}\left(R_{(t)}-\widehat{E}(R \mid \text { data })\right)^{2}$. 
Employing $T$ and $R$ values and utilizing the technique presented by Chen

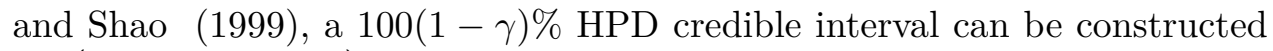
as $\left(R_{\left[\frac{\gamma}{2} T\right]}, R_{\left[\left(1-\frac{\gamma}{2}\right) T\right]}\right)$, where $R_{\left[\frac{\gamma}{2} T\right]}$ and $R_{\left[\left(1-\frac{\gamma}{2}\right) T\right]}$ are the $\left[\frac{\gamma}{2} T\right]$-th smallest integer and the $\left[\left(1-\frac{\gamma}{2}\right) T\right]$-th smallest integer of $\left\{R_{(t)}=1,2, \ldots, T\right\}$, respectively.

\section{Estimation of $R$ in Case of Known $\lambda$}

Now the estimation of $R$ in case of known $\lambda$ is presented.

\subsection{Maximum Likelihood Estimation of $\boldsymbol{R}$}

Let $X \sim K u(\alpha, \lambda)$ and $Y \sim K u(\beta, \lambda)$ be independent random variables. The purpose is estimating $R$ for progressive Type-II censored data drawn from both random variables when the common second shape parameter of $\mathrm{KuD}$ is known. Based on Section 3, it is clear that the MLE of $R$ will be $\widehat{R}=\frac{\widehat{\alpha}}{\widehat{\alpha}+\widehat{\beta}}$, where

$$
\widehat{\alpha}=-\frac{n}{\sum_{i=1}^{n}\left(R_{i}+1\right) \log \left(1-x_{i}^{\lambda}\right)}, \quad \widehat{\beta}=-\frac{m}{\sum_{j=1}^{m}\left(S_{j}+1\right) \log \left(1-y_{j}^{\lambda}\right)} .
$$

Therefore

$$
\widehat{R}_{M L}=\frac{n \sum_{j=1}^{m}\left(S_{j}+1\right) \log \left(1-y_{j}^{\lambda}\right)}{n \sum_{j=1}^{m}\left(S_{j}+1\right) \log \left(1-y_{j}^{\lambda}\right)+m \sum_{i=1}^{n}\left(R_{i}+1\right) \log \left(1-x_{i}^{\lambda}\right)} .
$$

With the same method in Section $2.2, \sqrt{n}\left(\widehat{R}_{M L}-R\right) \stackrel{D}{\longrightarrow} N(0, C)$ where

$$
C=\frac{\alpha^{2} \beta^{2}(1+p)}{(\alpha+\beta)^{4}}
$$

in which $p$ is the limit of $n / m$. Hence, a $100(1-\gamma) \%$ asymptotic confidence interval for $R$ is as $\left(\widehat{R}_{M L}-z_{1-\frac{\gamma}{2}} \frac{\sqrt{\widehat{C}}}{\sqrt{n}}, \widehat{R}_{M L}+z_{1-\frac{\gamma}{2}} \frac{\sqrt{\widehat{C}}}{\sqrt{n}}\right)$, where $z_{\gamma}$ is $100 \gamma$-th percentile of $N(0,1)$. Based on the asymptotic distribution of $R$, the Boot-p and Boot-t intervals of $R$ can be presented. As the bootstrap procedures are very similar to those of indicated in Section 2.3, we omit them. We remind that in Boot-p and Boot-t methods, $\widehat{R}^{*}$ and $V\left(\widehat{R}^{*}\right)$, when $\lambda$ is known are obtained from (6) and (7), respectively. 


\section{$3.2 \quad$ UMVUE of $R$}

In this section, we derive the UMVUE of $R$. Let $\left\{X_{1: n: N}, \ldots, X_{n: n: N}\right\}$ and $\left\{Y_{1: m: M}, \ldots, Y_{m: m: M}\right\}$ be two progressively censored samples from $K u(\alpha, \lambda)$ and $K u(\beta, \lambda)$ under the progressive schemes $\left\{N, n, R_{1}, R_{2}, \ldots, R_{n}\right\}$ and $\left\{Y_{1: m: M}, \ldots, Y_{m: m: M}\right\}$, respectively. The joint pdf of $X_{1: n: N}, \ldots, X_{n: n: N}$ is

$$
f_{X_{1: n: N}, \ldots, X_{n: n: N}}\left(x_{1}, \ldots, x_{n}\right)=c_{1}(\alpha \lambda)^{n}\left(\prod_{i=1}^{n} \frac{x_{i}^{\lambda-1}}{1-x_{i}^{\lambda}}\right) e^{\alpha \sum_{i=1}^{n}\left(R_{i}+1\right) \log \left(1-x_{i}^{\lambda}\right)}
$$

where $0<x_{1}<\ldots<x_{n}<1$. It is immediate from (8) that $U=$ $-\sum_{i=1}^{n}\left(R_{i}+1\right) \log \left(1-x_{i}^{\lambda}\right)$ is the complete sufficient statistics for $\alpha$ when $\lambda$ is known. It is clear that

$$
X_{i: n: N}^{*}=-\log \left(1-X_{i: n: N}^{\lambda}\right), \quad i=1, \ldots, n,
$$

be a progressive censored sample from an exponential distribution with mean $\alpha^{-1}$. Let

$$
\begin{aligned}
Z_{1} & =N X_{1: n: N}^{*}, \\
Z_{2} & =\left(N-R_{1}-1\right)\left(X_{2: n: N}^{*}-X_{1: n: N}^{*}\right), \\
\vdots & \\
Z_{n} & =\left(N-R_{1}-\ldots-R_{n-1}-n+1\right)\left(X_{n: n: N}^{*}-X_{n-1: n: N}^{*}\right) .
\end{aligned}
$$

From Cao and Cheng (2006), we get that $Z_{1}, \ldots, Z_{n}$ are independent and identically distributed exponential random variables with mean $\alpha^{-1}$. Moreover $U=\sum_{i=1}^{n} Z_{i}=\sum_{i=1}^{n}\left(R_{i}+1\right) X_{i: n: N}^{*}$ has a gamma distribution with the shape parameter $n$ and the scale parameter $\alpha$, in symbols $U \sim \Gamma(n, \alpha)$, i.e. it has the pdf

$$
f_{U}(u)=\frac{\alpha^{n}}{\Gamma(n)} u^{n-1} e^{-\alpha u}, \quad u>0
$$

Lemma 1. Let $Y_{j: m: M}^{*}=-\log \left(1-Y_{j: m: M}^{\lambda}\right)$ and $V=\sum_{j=1}^{m}\left(S_{j}+1\right) Y_{j: m: M}^{*}$ .The conditional pdf of $X_{1: n: N}^{*}$ given $U=u$, is

$$
f_{X_{1: n: N}^{*} \mid U=u}(x)=N(n-1) \frac{(u-N x)^{n-2}}{u^{n-1}}, \quad 0<x<u / N,
$$


and the conditional pdf of $Y_{1: m: M}^{*}$ given $V=v$, is

$$
f_{Y_{1: m: M}^{*} \mid V=v}(y)=M(m-1) \frac{(v-M y)^{m-2}}{v^{m-1}}, \quad 0<y<v / M .
$$

Proof. Here, we prove the first part, the second part follows a similar procedure to be proved. Note that

$$
f_{X_{1: n: N}^{*} \mid U=u}(x)=\frac{f_{X_{1: n: N}^{*}, U}(x, u)}{f_{U}(u)},
$$

where $f_{X_{1: n: N}^{*}, U}(x, u)$ is the joint pdf of $X_{1: n: N}^{*}$ and $U$ and $f_{U}(u)$ is the pdf of $U$. When $\lambda$ is known, $U$ is a complete sufficient statistics for $\alpha$. Suppose we denote $W=\sum_{i=2}^{n} Z_{i}$ then clearly $W$ and $Z_{1}$ are independent. The joint pdf of $X_{1: n: N}^{*}$ and $U$ can be easily obtained from the joint pdf of $W$ and $Z_{1}$, using the the transformation $Z_{1}=N X_{1: n: N}^{*}$ and $U=W+Z_{1}$. Finally the result is found using (9).

Theorem 3. The UMVUE of $R, \tilde{R}$, is:

$$
\tilde{R}=\left\{\begin{array}{c}
1-\sum_{k=0}^{n-1}(-1)^{k}\left(\frac{v}{u}\right)^{k} \frac{\left(\begin{array}{c}
n-1 \\
k
\end{array}\right)}{\left(\begin{array}{c}
m+k-1 \\
k
\end{array}\right)} \quad \text { if } v<u, \\
\sum_{k=0}^{m-1}(-1)^{k}\left(\frac{u}{v}\right)^{k} \frac{\left(\begin{array}{c}
m-1 \\
k
\end{array}\right)}{\left(\begin{array}{c}
n+k-1 \\
k
\end{array}\right)} \quad \text { if } v>u .
\end{array}\right.
$$

where $n, m \geq 2$, and $U$ and $V$ are respectively complete sufficient statistics for $\alpha$ and $\beta$, as mentioned before.

Proof. See Appendix B.

\subsection{Exact Confidence Interval}

In this section, we derive the exact confidence interval of $R$. So, let $\left\{X_{1: n: N}\right.$, $\left.\ldots, X_{n: n: N}\right\}$ and $\left\{Y_{1: m: M}, \ldots, Y_{m: m: M}\right\}$ be a progressively censored sample from $K u(\alpha, \lambda)$ and $K u(\beta, \lambda)$ under the progressive censoring scheme $\left\{N, n, R_{1}, R_{2}, \ldots, R_{n}\right\}$ and $\left\{Y_{1: m: M}, \ldots, Y_{m: m: M}\right\}$, respectively. Let $X_{i: n: N}^{* *}=$ $-\alpha \log \left(1-X_{i: n: N}^{\lambda}\right), i=1, \ldots, n\left(\right.$ or $Y_{j: m: M}^{* *}=-\beta \log \left(1-Y_{j: m: M}^{\lambda}\right), j=$ $1, \ldots, m)$. It is easy to see that $X_{i: n: N}^{* *}, i=1, \ldots, n\left(\right.$ or $Y_{j: m: M}^{* *}, j=$ 
$1, \ldots, m)$ is a progressive censoring sample from a standard exponential distribution. Let us consider the following transformations:

$$
\begin{aligned}
& Z_{1}^{*}=N X_{1: n: N}^{* *}, Z_{i}^{*}=\left[N-\sum_{k=1}^{i-1}\left(R_{k}+1\right)\right]\left(X_{i: n: N}^{* *}-X_{i-1: n: N}^{* *}\right), i=2, \ldots, n, \\
& D_{1}^{*}=M Y_{1: m: M}^{* *}, D_{j}^{*}=\left[M-\sum_{k=1}^{j-1}\left(S_{k}+1\right)\right]\left(Y_{j: m: M}^{* *}-Y_{j-1: m: M}^{* *}\right), j=2, \ldots, m .
\end{aligned}
$$

From Cao and Cheng (2006), we get that $Z_{1}^{*}, \ldots, Z_{n}^{*}$ (or $D_{1}^{*}, \ldots, D_{m}^{*}$ ) are independent and identically distribution as standard exponential.

Let $V_{X}=2 Z_{1}^{*}$ and $U_{X}=2 \sum_{i=2}^{n} Z_{i}^{*}$ (or $V_{Y}=2 D_{1}^{*}$ and $U_{Y}=2 \sum_{j=2}^{m} D_{j}^{*}$ ). Then $V_{X}$ and $U_{X}$ (or $V_{Y}$ and $U_{Y}$ ) are independent random variables and

$$
V_{X} \sim \chi_{(2)}^{2} \text { and } U_{X} \sim \chi_{(2 n-2)}^{2}\left(\text { or } V_{Y} \sim \chi_{(2)}^{2} \text { and } U_{Y} \sim \chi_{(2 m-2)}^{2}\right) .
$$

Lemma 2. Let $T_{X}(\lambda)=\frac{U_{X}}{(n-1) V_{X}}, T_{1}=U_{X}+V_{X}, T_{Y}(\lambda)=\frac{U_{Y}}{(m-1) V_{Y}}$ and $T_{2}=U_{Y}+V_{Y}$. We can find that

$T_{X}(\lambda) \sim F(2 n-2,2), T_{1} \sim \chi^{2}(2 n), T_{Y}(\lambda) \sim F(2 m-2,2)$ and $T_{2} \sim \chi^{2}(2 m)$.

It is obvious that $T_{X}(\lambda)$ and $T_{Y}(\lambda)$ are independent. Furthermore, Johnson, et al. (1994) indicated that $T_{X}(\lambda)$ and $T_{1}\left(\right.$ or $T_{Y}(\lambda)$ and $\left.T_{2}\right)$ are independent.

Lemma 3. $T_{X}(\lambda)$ (or $T_{Y}(\lambda)$ ) is strictly decreasing in $\lambda$.

Proof. See Appendix C.

Lemma 4. When $\lambda$ is known, the MLE of $R$ can be obtained as

$$
\widehat{R}_{M L}=\frac{1}{1+\frac{m T_{1} / \alpha}{n T_{2} / \beta}} .
$$

It is obvious that $T_{1}$ and $T_{2}$ are independent. Alternatively, $T_{X}(\lambda), T_{Y}(\lambda)$ and $\widehat{R}_{M L}$ are independent. Also, by using Lemma 2, we have

$$
\widehat{R}_{M L}=\frac{1}{1+\frac{\beta}{\alpha} F}, \quad \text { or } \quad F=\frac{R}{1-R} \cdot \frac{1-\widehat{R}_{M L}}{\widehat{R}_{M L}},
$$


where $F$ has Fisher distribution with $(2 n, 2 m)$ degrees of freedom, in symbols $F \sim F(2 n, 2 m)$.

Theorem 4. Suppose that $\left\{X_{1: n: N}, \ldots, X_{n: n: N}\right\}$ be a progressively censored sample from $K u(\alpha, \lambda)$ and $\left\{Y_{1: m: M}, \ldots, Y_{m: m: M}\right\}$ be a progressively censored sample from $K u(\beta, \lambda)$. Then

(i) for any $0<\gamma<1$,

$$
\begin{aligned}
& \left(\max \left\{T_{X}^{-1}\left(F_{(1+\sqrt{1-\gamma}) / 2}(2 n-2,2)\right), T_{Y}^{-1}\left(F_{(1+\sqrt{1-\gamma}) / 2}(2 m-2,2)\right)\right\},\right. \\
& \left.\min \left\{T_{X}^{-1}\left(F_{(1-\sqrt{1-\gamma}) / 2}(2 n-2,2)\right), T_{Y}^{-1}\left(F_{(1-\sqrt{1-\gamma}) / 2}(2 m-2,2)\right)\right\}\right)
\end{aligned}
$$

is a $100(1-\gamma) \%$ confidence interval for $\lambda$, where $F_{\gamma}(p, q)$ is $100 \gamma$-th percentile of $F(p, q)$.

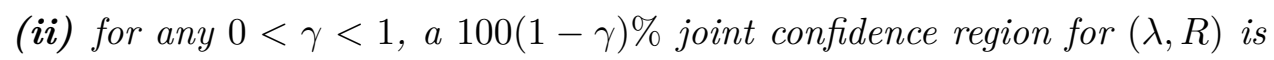
determined by the following inequalities

$$
\left\{\begin{array}{c}
\max \left\{T_{X}^{-1}\left(F_{(1+\sqrt[4]{1-\gamma}) / 2}(2 n-2,2)\right), T_{Y}^{-1}\left(F_{(1+\sqrt[4]{1-\gamma}) / 2}(2 m-2,2)\right)\right\} \leq \lambda \\
\leq \min \left\{T_{X}^{-1}\left(F_{(1-\sqrt[4]{1-\gamma}) / 2}(2 n-2,2)\right), T_{Y}^{-1}\left(F_{(1-\sqrt[4]{1-\gamma}) / 2}(2 m-2,2)\right)\right\} \\
\frac{1}{1+\frac{1-\widehat{R}_{M L}}{\widehat{R}_{M L}} F_{1-(1-\sqrt{1-\gamma}) / 2}(2 m, 2 n)} \leq R \leq \frac{1}{1+\frac{1-\widehat{R}_{M L}}{\widehat{R}_{M L}} F_{1-(1+\sqrt{1-\gamma}) / 2}(2 m, 2 n)}
\end{array}\right.
$$

Proof. See Appendix D.

\subsection{Bayes Estimation of $\mathrm{R}$}

Assuming that $\lambda$ is known and $\alpha$ and $\beta$ are both random variables, we derive Bayes estimation of $R$ in this section. Moreover, we consider two independent gamma distributions with the parameters $\left(a_{1}, b_{1}\right)$ and $\left(a_{2}, b_{2}\right)$ as two priors for $\alpha$ and $\beta$, respectively. It can be shown that $\alpha$ and $\beta$ have the posterior pdfs $\Gamma\left(n+a_{1}, b_{1}+A_{1}(\mathbf{x})\right)$ and $\Gamma\left(m+a_{2}, b_{2}+A_{2}(\mathbf{y})\right)$, respectively, where $\left.A_{1}(\mathbf{x})\right)=T_{1} /(2 \alpha)$ and $\left.A_{2}(\mathbf{y})\right)=T_{2} /(2 \beta)$. Considering the independence of 
priors, the posterior pdf of $R$ turn out to be

$$
f_{R}(r)=S \frac{r^{n+a_{1}-1}(1-r)^{m+a_{2}-1}}{\left[r\left(b_{1}+A_{1}(\mathbf{x})\right)+(1-r)\left(b_{2}+A_{2}(\mathbf{y})\right)\right]^{n+m+a_{1}+a_{2}}}, \quad 0<r<1,
$$

where

$$
S=\frac{\Gamma\left(n+m+a_{1}+a_{2}\right)}{\Gamma\left(n+a_{1}\right) \Gamma\left(m+a_{2}\right)}\left(b_{1}+A_{1}(\mathbf{x})\right)^{n+a_{1}}\left(b_{2}+A_{2}(\mathbf{y})\right)^{m+a_{2}} .
$$

Under the squared error loss function, we are not able to obtain a closed form for the Bayes estimation of $R$, so it would be approximated by Lindley method Lindley (1980). Alternatively, utilizing the approximation of Lindley (1980) and the idea of Ahmad, et al. (1997), under the squared error loss function the Bayes estimation of $R, \widehat{R}_{B S}$, can be approximated by

$$
\widehat{R}_{B S}=\breve{R}\left\{1+\frac{\breve{\alpha} \breve{R}^{2}}{\breve{\beta}^{2}\left(m+a_{2}-1\right)\left(n+b_{1}-1\right)}\left[\breve{\alpha}\left(n+a_{1}-1\right)-\breve{\beta}\left(m+a_{2}-2\right)\right]\right\},
$$

where

$$
\breve{R}=\frac{\breve{\alpha}}{\breve{\alpha}+\breve{\beta}}, \quad \breve{\alpha}=\frac{n+a_{1}-1}{b_{1}+A_{1}(\mathbf{x})}, \quad \text { and } \quad \breve{\beta}=\frac{m+a_{2}-1}{b_{2}+A_{2}(\mathbf{y})}
$$

The $100(1-\gamma) \%$ Bayesian interval for $R$ is $(L, U)$ in which $L$ is the lower and $U$ is the upper bound, satisfying

$$
P[R \leq L \mid \text { data }]=\frac{\gamma}{2}, \quad \text { and } \quad P[R \leq U \mid \text { data }]=1-\frac{\gamma}{2} .
$$

\section{Estimation of $R$ in General Case}

Now, we investigate estimations of $R$ in case of different shape parameters.

\subsection{Maximum Likelihood Estimation of $R$}

Let $X \sim K u\left(\alpha, \lambda_{1}\right)$ and $Y \sim K u\left(\beta, \lambda_{2}\right)$ be independent random variables. Therefore, 


$$
\begin{aligned}
R=P(X<Y) & =\int_{0}^{1} f_{Y}(y) P(X<Y \mid Y=y) d y \\
& =\int_{0}^{1} \beta \lambda_{2} y^{\lambda_{2}-1}\left(1-y^{\lambda_{2}}\right)^{\beta-1}\left(1-\left(1-y^{\lambda_{1}}\right)^{\alpha}\right) d y \\
& =1-\int_{0}^{1} \beta \lambda_{2} y^{\lambda_{2}-1}\left(1-y^{\lambda_{2}}\right)^{\beta-1}\left(1-y^{\lambda_{1}}\right)^{\alpha} d y
\end{aligned}
$$

Obviously, $R$ cannot be expressed in a closed form,accordingly numerical computation have to be performed for any inference about $R$. To obtain the maximum likelihood estimation of $R$, we proceed as follows. Let $\left\{X_{1: n: N}, \ldots\right.$, $\left.X_{n: n: N}\right\}$ and $\left\{Y_{1: m: M}, \ldots, Y_{m: m: M}\right\}$ be a progressively censored sample from $K u(\alpha, \lambda)$ and $K u(\beta, \lambda)$ under the progressive censoring scheme $\left\{N, n, R_{1}, R_{2}\right.$, $\left.\ldots, R_{n}\right\}$ and $\left\{Y_{1: m: M}, \ldots, Y_{m: m: M}\right\}$, respectively. Therefore, the log-likelihood function of the observed data is

$$
\begin{aligned}
\ell\left(\alpha, \beta, \lambda_{1}, \lambda_{2}\right)= & n \log (\alpha)+m \log (\beta)+n \log \left(\lambda_{1}\right) \\
& +m \log \left(\lambda_{2}\right)+\left(\lambda_{1}-1\right) \sum_{i=1}^{n} \log \left(x_{i}\right) \\
& +\left(\lambda_{2}-1\right) \sum_{j=1}^{m} \log \left(y_{j}\right)+\sum_{i=1}^{n}\left(\alpha\left(R_{i}+1\right)-1\right) \log \left(1-x_{i}^{\lambda_{1}}\right) \\
& +\sum_{j=1}^{m}\left(\beta\left(S_{j}+1\right)-1\right) \log \left(1-y_{j}^{\lambda_{2}}\right)+\text { Constant. }
\end{aligned}
$$

The MLEs of $\alpha$ and $\beta$ say $\widehat{\alpha}$ and $\widehat{\beta}$, respectively, are

$$
\widehat{\alpha}\left(\lambda_{1}\right)=-\frac{n}{\sum_{i=1}^{n}\left(R_{i}+1\right) \log \left(1-x_{i}^{\lambda_{1}}\right)}, \quad \widehat{\beta}\left(\lambda_{2}\right)=-\frac{m}{\sum_{j=1}^{m}\left(S_{j}+1\right) \log \left(1-y_{j}^{\lambda_{2}}\right)} .
$$

The MLEs of $\lambda_{1}$ and $\lambda_{2}$ say $\hat{\lambda}_{1}$ and $\hat{\lambda}_{2}$, are obtained by solving the following nonlinear equations 


$$
\begin{aligned}
& k_{1}\left(\lambda_{1}\right)=-n\left\{\sum_{i=1}^{n} \log \left(x_{i}\right)\left(-1+\frac{\left(\alpha\left(R_{i}+1\right)-1\right) x_{i}^{\lambda_{1}}}{1-x_{i}^{\lambda_{1}}}\right)\right\}^{-1}, \\
& k_{2}\left(\lambda_{2}\right)=-m\left\{\sum_{j=1}^{m} \log \left(y_{j}\right)\left(-1+\frac{\left(\beta\left(S_{j}+1\right)-1\right) y_{j}^{\lambda_{2}}}{1-y_{j}^{\lambda_{2}}}\right)\right\}^{-1} .
\end{aligned}
$$

Using the invariance property of the MLEs, the MLE of $R$ becomes

$$
R=1-\int_{0}^{1} \widehat{\beta} \widehat{\lambda}_{2} y^{\widehat{\lambda}_{2}-1}\left(1-y^{\widehat{\lambda}_{2}}\right)^{\widehat{\beta}-1}\left(1-y^{\widehat{\lambda}_{1}}\right)^{\widehat{\alpha}} d y
$$

\subsection{Asymptotic Confidence Interval}

Similar to the approach in Section 2.2, the asymptotic distribution of $\widehat{\theta}=$ $\left(\widehat{\alpha}, \widehat{\beta}, \widehat{\lambda}_{1}, \widehat{\lambda}_{2}\right)$ is obtained in this section. The expected Fisher information matrix of $\theta=\left(\alpha, \beta, \lambda_{1}, \lambda_{2}\right)$ is denoted as $J(\theta)=-E(I(\theta))$, in which $I(\theta)=$ $\left[I_{i j}\right], i, j=1,2,3,4$ is the observed information matrix. The elements of $J(\theta)$ and asymptotic normality of the MLEs are given in Appendix A.

\subsection{Bayes Estimation of $R$}

Now assuming that the parameters $\alpha, \beta, \lambda_{1}$ and $\lambda_{2}$ are random variables, we draw the Bayesian inference of $R$. The Bayes estimations and the associated credible intervals of $R$ are mainly discussed. Let suppose the priors of $\alpha, \beta$, $\lambda_{1}$ and $\lambda_{2}$ are independent gamma distributions as:

$$
\begin{aligned}
\pi_{1}(\alpha) & \propto \alpha^{a_{1}-1} e^{-b_{1} \alpha}, & & \alpha>0, a_{1}>0, b_{1}>0, \\
\pi_{2}(\beta) & \propto \beta^{a_{2}-1} e^{-b_{2} \beta}, & & \beta>0, a_{2}>0, b_{2}>0, \\
\pi_{3}\left(\lambda_{1}\right) & \propto \lambda_{1}^{a_{3}-1} e^{-b_{3} \lambda_{1}}, & & \lambda_{1}>0, a_{3}>0, b_{3}>0, \\
\pi_{4}\left(\lambda_{2}\right) & \propto \lambda_{2}^{a_{4}-1} e^{-b_{4} \lambda_{2}}, & & \lambda_{2}>0, a_{4}>0, b_{4}>0,
\end{aligned}
$$

respectively. The reasons, for choosing the gamma priors, can be explained as follows. The range of $\alpha, \beta, \lambda_{1}, \lambda_{2}$ are positive. Also, because the full conditional distributions of $\alpha$ and $\beta$, given $\lambda_{1}, \lambda_{2}$ and data, are gamma distributions, so, they are conjugated priors. Finally, by selecting these gamma 
priors, the calculations become a little easier. The joint posterior density function of $\alpha, \beta, \lambda_{1}$ and $\lambda_{2}$ is defined by

$$
\begin{aligned}
& \pi\left(\alpha, \beta, \lambda_{1}, \lambda_{2} \mid \text { data }\right)= \\
& \frac{L\left(\text { data } \mid \alpha, \beta, \lambda_{1}, \lambda_{2}\right) \pi_{1}(\alpha) \pi_{2}(\beta) \pi_{3}\left(\lambda_{1}\right) \pi_{4}\left(\lambda_{2}\right)}{\int_{0}^{\infty} \int_{0}^{\infty} \int_{0}^{\infty} \int_{0}^{\infty} L\left(\text { data } \mid \alpha, \beta, \lambda_{1}, \lambda_{2}\right) \pi_{1}(\alpha) \pi_{2}(\beta) \pi_{3}\left(\lambda_{1}\right) \pi_{4}\left(\lambda_{2}\right) d \alpha d \beta d \lambda_{1} d \lambda_{2}} .
\end{aligned}
$$

It is obvious that the Bayes estimate will not be analytically computed. Therefore, to obtain the Bayes estimation and the associated credible interval of $R$, we employ the Gibbs and Metropolis sampling methods. The posterior pdfs of $\alpha$ and $\beta$ are:

$$
\begin{aligned}
& \alpha \mid \beta, \lambda_{1}, \lambda_{2}, \text { data } \sim \Gamma\left(n+a_{1}, b_{1}+\sum_{i=1}^{n}\left(R_{i}+1\right) \log \left(\frac{1}{1-x_{i}^{\lambda_{1}}}\right)\right), \\
& \beta \mid \alpha, \lambda_{1}, \lambda_{2}, \text { data } \sim \Gamma\left(m+a_{2}, b_{2}+\sum_{j=1}^{m}\left(S_{j}+1\right) \log \left(\frac{1}{1-y_{j}^{\lambda_{2}}}\right)\right),
\end{aligned}
$$

and

$$
\begin{aligned}
& \pi\left(\lambda_{1} \mid \alpha, \beta, \lambda_{2}, \text { data }\right) \propto \lambda_{1}^{n+a_{3}-1} e^{-b_{3} \lambda_{1}}\left(\prod_{i=1}^{n} \frac{x_{i}^{\lambda_{1}-1}}{1-x_{i}^{\lambda_{1}}}\right), \\
& \pi\left(\lambda_{2} \mid \alpha, \beta, \lambda_{1}, \text { data }\right) \propto \lambda_{2}^{m+a_{4}-1} e^{-b_{4} \lambda_{2}}\left(\prod_{j=1}^{m} \frac{y_{j}^{\lambda_{2}-1}}{1-y_{j}^{\lambda_{2}}}\right) .
\end{aligned}
$$

Because $\lambda_{1}$ and $\lambda_{2}$ have the unknown posterior pdfs, for generating random numbers from posterior pdfs of $\lambda_{1}$ and $\lambda_{2}$ the Metropolis-Hastings approach is employed. Then, the Gibbs sampling's algorithm would be:

1. Begin with an initial guess $\left(\alpha_{(0)}, \beta_{(0)}, \lambda_{1(0)}, \lambda_{2(0)}\right)$.

2. Set $t=1$.

3. Generate $\lambda_{1(t)}$ from $\pi\left(\lambda_{1} \mid \alpha_{(t-1)}, \beta_{(t-1)}, \lambda_{2(t-1)}\right.$, data).

4. Generate $\lambda_{2(t)}$ from $\pi\left(\lambda_{2} \mid \alpha_{(t-1)}, \beta_{(t-1)}, \lambda_{1(t-1)}\right.$, data).

5. Generate $\alpha_{(t)}$ from $\Gamma\left(n+a_{1}, b_{1}+\sum_{i=1}^{n}\left(R_{i}+1\right) \log \left(\frac{1}{1-x_{i}^{\lambda_{1}(t-1)}}\right)\right)$. 
6. Generate $\beta_{(t)}$ from $\Gamma\left(m+a_{2}, b_{2}+\sum_{j=1}^{m}\left(S_{j}+1\right) \log \left(\frac{1}{1-y_{j}^{\lambda_{2(t-1)}}}\right)\right)$.

7. Compute $R_{(t)}=1-\int_{0}^{1} \beta_{(t)} \lambda_{2(t)} y^{\lambda_{2(t)}-1}\left(1-y^{\lambda_{2(t)}}\right)^{\beta_{(t)}-1}\left(1-y^{\lambda_{1(t)}}\right)^{\alpha_{(t)}} d y$.

8. Set $t=t+1$.

9. Iterate $3-8, T$ times.

Accordingly, the mean and the variance of posterior of $R$ are approximately given by

$\widehat{E}(R \mid$ data $)=\frac{1}{T} \sum_{t=1}^{T} R_{(t)}, \widehat{\operatorname{Var}}(R \mid$ data $)=\frac{1}{T} \sum_{t=1}^{T}\left(R_{(t)}-\widehat{E}(R \mid \text { data })\right)^{2}$.

Employing the technique presented by Chen and Shao (1999), and using $T$ and $R$ values a $100(1-\gamma) \%$ HPD credible interval can be constructed as $\left(R_{\left[\frac{\gamma}{2} T\right]}, R_{\left[\left(1-\frac{\gamma}{2}\right) T\right]}\right)$, where $R_{\left[\frac{\gamma}{2} T\right]}$ and $R_{\left[\left(1-\frac{\gamma}{2}\right) T\right]}$ are the $\left[\frac{\gamma}{2} T\right]$-th smallest integer and the $\left[\left(1-\frac{\gamma}{2}\right) T\right]$-th smallest integer of $\left\{R_{(t)}=1,2, \ldots, T\right\}$, respectively.

\section{Data Analysis and Comparison Study}

For comparative purposes, some experiments are conducted in this section using Monte Carlo methods and real data analysis.

\subsection{Numerical Results and Discussions}

Now, considering different progressive censoring schemes we make some Monte Carlo simulations for performance comparison of different methods. Three cases have been considered here to draw inference on $R$. First case is assuming unknown common shape parameter $\lambda$. Second, the common shape parameter $\lambda$ is assumed known. Third the second shape parameters $\lambda_{1}$ and $\lambda_{2}$ are both unknown.

For the first case, that is the unknown common second shape parameter $\lambda$, we compare performances of the presented estimations in terms of the mean square errors (MSE). Moreover, different confidence intervals are compared together in the sense of average confidence lengths, and coverage probabilities. Different parameter values, hyper parameters and censored 
sampling schemes have been used in the simulations. Four sets of parameter values are used to evaluate the ML and Bayes estimators that are $\Theta_{1}=(\alpha=$ $2, \beta=2, \lambda=1.5), \Theta_{2}=(\alpha=2, \beta=2, \lambda=2), \Theta_{3}=(\alpha=2, \beta=2, \lambda=2.5)$ and $\Theta_{4}=(\alpha=3.7597, \beta=1.9016, \lambda=2.5852)$. It is notable that $\Theta_{4}$ is the values to the estimated parameters of the real data which considered in the Section 5.2. Three priors are assumed to evaluate the Bayes estimations and HPD intervals that are

$\begin{array}{llll}\text { Prior 1: } & a_{j}=0, & b_{j}=0, & j=1,2,3, \\ \text { Prior 2: } & a_{j}=1, & b_{j}=2, & j=1,2,3, \\ \text { Prior 3: } & a_{j}=2, & b_{j}=3, & j=1,2,3 .\end{array}$

Prior 1 is a non-informative prior and Prior 2 and Prior 3 are informative ones. Furthermore, three censored sampling schemes are employed based on the following:

$$
\begin{aligned}
& r_{1}: R_{2 k}=\frac{N-n}{n}-1, R_{2 k-1}=\frac{N-n}{n}-1, k=1, \ldots, \frac{n}{2}, \\
& r_{2}: R_{2 k}=\frac{2(N-n)}{n}, R_{2 k-1}=0, \quad k=1, \ldots, \frac{n}{2}, \\
& r_{3}: R_{1}=\cdots=R_{n}=\frac{N-n}{n} .
\end{aligned}
$$

To see the effect of censoring scheme on the precision of the stress-strength parameter, we obtain the results based on the above schemes and $(n, N)=$ $(10,40),(20,40)$. The average biases, and MSEs of different estimators of $R$ over 1000 repetitions are reported in Table 1 . In the simulations of bootstrap methods, we have used 250 re-sampling to compute the confidence intervals. Considering 1000 sampling $(T=1000)$, the Bayes estimations and the associated credible intervals have been obtained. Comparing MSEs in Table 1 indicates performance of ML estimator is comparable to the Bayes estimator. Also, we observe that the Bayes estimates perform better than the ML estimates. Moreover, in Bayesian estimates, informative Priors 2 and 3 have better performance than non-informative ones, so that Prior 3 is the best estimate in terms of MSEs. Moreover, the $95 \%$ asymptotic confidence intervals of $R$ have been computed in the sense of asymptotic distribution of the ML estimation. We also computed the Boot-p, Boot-t and the HPD credible intervals. The experimental results are presented in Table 2. In all cases, the nominal level of 0.95 for the confidence intervals is considered. From Table 2, it is observed that the bootstrap confidence intervals are the worst 
confidence intervals, so that they have the largest average lengths and smallest coverage probabilities. Also, comparing different parameter values and censored sampling methods shows the HPD credible intervals have the best performance based on average lengths and coverage probabilities. Moreover, in Bayesian inference, the performance of HPD credible intervals obtained by the informative priors is better than non-informative ones, so that the smallest average lengths with the largest coverage probabilities belongs to Prior 3. The second best confidence interval is the asymptotic confidence interval of MLE. In addition, when we calculated the $T^{*}$ statistics, we could not do re-center the bootstrap resamples. So, it is observed that Boot-p confidence intervals perform better than the Boot-t confidence intervals (see Baklizi (2007), for more details).

Considering the case that the common second shape parameter is known, the ML and UMVU estimations of $R$ using (6) and (10) are obtained. We also provide the asymptotic confidence intervals and two non-parametric bootstrap intervals. As there is no prior information about $R$, for computing the Bayes estimates using the non-informative prior i.e $a_{1}=b_{1}=a_{2}=b_{2}=0$ is preferred. We obtain Bayes estimations by implementing the same prior distributions, based on Lindley approximation in accordance with (11). Furthermore, using (12) the Bayesian interval based on Lindley approximation is obtained. The average biases and MSEs resulted from 1000 repetitions are stated in Table 3. According to this table, we observe that Bayes estimate has the best performance, based on MSEs and the MSEs of UMVUE is greater than the MLE. As we know, the UMVUE gives the best estimator in the class of unbiased estimators. But, in the case of study, MLE tries to minimize the MSE which is the sum of squared bias and variance of the estimator. Thus, the MLE has lower MSE value than the UMVUE since it has significant decrease in the variance as compared to the increment in the bias value. Comparing different confidence intervals shows that the bootstrap and Bayesian intervals are the worst and best confidence intervals, respectively, based on the average lengths and coverage probabilities. The second best confidence interval is the asymptotic confidence interval of MLE. In addition, it is observed that Boot-p confidence intervals perform better than the Boot-t confidence intervals.

In the third case when $\lambda_{1}$ and $\lambda_{2}$ are different and unknown, $R$ is estimated using the MLE and Bayes approaches. To compare the MLEs and Bayes estimations, four sets of parameter values are used as $\Theta_{5}=(\alpha=$ $\left.2, \beta=2, \lambda_{1}=1.5, \lambda_{2}=1.5\right), \Theta_{6}=\left(\alpha=2, \beta=2, \lambda_{1}=1.5, \lambda_{2}=2\right)$, 
$\Theta_{7}=\left(\alpha=2, \beta=2, \lambda_{1}=1.5, \lambda_{2}=2.5\right)$ and $\Theta_{8}=(\alpha=3.5653, \beta=$ $\left.2.0019, \lambda_{1}=2.4854, \lambda_{2}=2.7233\right)$. It is notable that $\Theta_{8}$ is the values to the estimated parameters of the real data which considered in the Section 5.2. The Bayes estimations and HPD credible intervals are computed based on three priors as follows:

$\begin{array}{llll}\text { Prior } 4: & a_{j}=0, & b_{j}=0, & j=1,2,3,4, \\ \text { Prior 5: } & a_{j}=1, & b_{j}=2, & j=1,2,3,4, \\ \text { Prior 6: } & a_{j}=2, & b_{j}=3, & j=1,2,3,4 .\end{array}$

In Table 4, we bring the average biases and MSEs of the different estimations of $R$ obtained over 1000 repetitions. In this table, the average lengths, and the associated coverage probabilities are presented. The nominal level for the credible intervals is 0.95 . According to Table 4, the MLE is comparable to the Bayes estimation in the sense of MSEs. Moreover, in Bayesian estimates, informative Priors 5 and 6 have better performance than non-informative ones, so that Prior 6 is the best estimate in terms of MSEs. Comparing the HPD credible intervals presents that the performance of HPD intervals obtained by the informative priors is better than non-informative ones, so that the smallest average lengths with the largest coverage probabilities belongs to Prior 6 .

As a fact, form Tables 1, 3 and 4 it is observed that, in all cases, for fixed $N$, with increasing $n$, in all cases, the MSEs of all estimations decrease. The reason of this is that with the decreasing amount of censored data, more information is gathered, and it makes the results more accurate. Furthermore, from Tables 2, 3 and 4, for fixed $N$, with increasing $n$, it is observed that, in all cases, the coverage probabilities increase, and the corresponding average confidence lengths decrease.

\subsection{Data Analysis}

Here, an analysis on a real strength data set, which was reported in Badar and Priest (1982) by Badar and Priest, is brought. In this data set, strengths of single carbon fibers, which measured in GPA, are presented. Single fibers were tested under tension at gauge lengths of $10 \mathrm{~mm}$ (Data Set 1) and $20 \mathrm{~mm}$ (Data Set 2). Several authors analyzed these data sets that are presented in Tables 5 and Table 6, respectively. By two independent works of Surles and Padgett (1998, 2001), Raqab and Kundu (2005), it has been shown that a generalized Rayleigh distribution performs quite well for these data 
Table 1. Biases and MSEs of the ML and Bayes estimations of $R$.

\begin{tabular}{|c|c|c|c|c|c|c|c|c|c|c|}
\hline \multirow[t]{2}{*}{$(n, N)$} & \multirow[t]{2}{*}{$\Theta_{j}$} & \multirow[t]{2}{*}{ C.S } & \multicolumn{2}{|c|}{ MLE } & \multicolumn{2}{|c|}{ Prior 1} & \multicolumn{2}{|c|}{ Prior 2} & \multicolumn{2}{|c|}{ Prior 3} \\
\hline & & & | Bias | & MSE & Bias | & MSE & | Bias | & MSE & Bias | & MSE \\
\hline \multirow{24}{*}{$(10,40)$} & \multirow{6}{*}{$\Theta_{1}$} & $\left(r_{1}, r_{1}\right)$ & 0.0026 & 0.0224 & 0.0065 & 0.0160 & 0.0028 & 0.0141 & 0.0030 & 0.0121 \\
\hline & & $\left(r_{2}, r_{2}\right)$ & 0.0060 & 0.0227 & 0.0097 & 0.0161 & 0.0092 & 0.0141 & 0.0036 & 0.0110 \\
\hline & & $\left(r_{3}, r_{3}\right)$ & 0.0002 & 0.0204 & 0.0046 & 0.0161 & 0.0052 & 0.0134 & 0.0027 & 0.0124 \\
\hline & & $\left(r_{1}, r_{2}\right)$ & 0.0032 & 0.0227 & 0.0031 & 0.0156 & 0.0035 & 0.0140 & 0.0089 & 0.0112 \\
\hline & & $\left(r_{1}, r_{3}\right)$ & 0.0090 & 0.0219 & 0.0017 & 0.0160 & 0.0042 & 0.0140 & 0.0058 & 0.0109 \\
\hline & & $\left(r_{2}, r_{3}\right)$ & 0.0084 & 0.0203 & 0.0078 & 0.0153 & 0.0034 & 0.0132 & 0.0042 & 0.0110 \\
\hline & \multirow{6}{*}{$\Theta_{2}$} & $\left(r_{1}, r_{1}\right)$ & 0.0089 & 0.0208 & 0.0081 & 0.0161 & 0.0039 & 0.0132 & 0.0053 & 0.0117 \\
\hline & & $\left(r_{2}, r_{2}\right)$ & 0.0048 & 0.0216 & 0.0076 & 0.0150 & 0.0086 & 0.0137 & 0.0076 & 0.0114 \\
\hline & & $\left(r_{3}, r_{3}\right)$ & 0.0046 & 0.0229 & 0.0064 & 0.0154 & 0.0049 & 0.0144 & 0.0021 & 0.0112 \\
\hline & & $\left(r_{1}, r_{2}\right)$ & 0.0015 & 0.0229 & 0.0048 & 0.0151 & 0.0055 & 0.0135 & 0.0010 & 0.0122 \\
\hline & & $\left(r_{1}, r_{3}\right)$ & 0.0010 & 0.0205 & 0.0019 & 0.0151 & 0.0034 & 0.0139 & 0.0008 & 0.0117 \\
\hline & & $\left(r_{2}, r_{3}\right)$ & 0.0089 & 0.0229 & 0.0095 & 0.0162 & 0.0069 & 0.0133 & 0.0032 & 0.0116 \\
\hline & \multirow{6}{*}{$\Theta_{3}$} & $\left(r_{1}, r_{1}\right)$ & 0.0016 & 0.0229 & 0.0015 & 0.0160 & 0.0031 & 0.0141 & 0.0054 & 0.0123 \\
\hline & & $\left(r_{2}, r_{2}\right)$ & 0.0097 & 0.0215 & 0.0037 & 0.0155 & 0.0056 & 0.0134 & 0.0030 & 0.0111 \\
\hline & & $\left(r_{3}, r_{3}\right)$ & 0.0040 & 0.0224 & 0.0068 & 0.0164 & 0.0035 & 0.0138 & 0.0032 & 0.0120 \\
\hline & & $\left(r_{1}, r_{2}\right)$ & 0.0040 & 0.0204 & 0.0064 & 0.0151 & 0.0099 & 0.0140 & 0.0017 & 0.0120 \\
\hline & & $\left(r_{1}, r_{3}\right)$ & 0.0033 & 0.0213 & 0.0015 & 0.0157 & 0.0020 & 0.0143 & 0.0068 & 0.0113 \\
\hline & & $\left(r_{2}, r_{3}\right)$ & 0.0008 & 0.0227 & 0.0081 & 0.0156 & 0.0023 & 0.0144 & 0.0067 & 0.0116 \\
\hline & \multirow{6}{*}{$\Theta_{4}$} & $\left(r_{1}, r_{1}\right)$ & 0.0040 & 0.0224 & 0.0020 & 0.0161 & 0.0083 & 0.0138 & 0.0049 & 0.0107 \\
\hline & & $\left(r_{2}, r_{2}\right)$ & 0.0033 & 0.0229 & 0.0006 & 0.0162 & 0.0100 & 0.0132 & 0.0023 & 0.0106 \\
\hline & & $\left(r_{3}, r_{3}\right)$ & 0.0064 & 0.022 & 0.0039 & 0.0153 & 0.0008 & 0.0132 & 0.0016 & 0.0116 \\
\hline & & $\left(r_{1}, r_{2}\right)$ & 0.0074 & 0.0201 & 0.0040 & 0.0157 & 0.0015 & 0.0134 & 0.0008 & 0.0121 \\
\hline & & $\left(r_{1}, r_{3}\right)$ & 0.0100 & 0.0225 & 0.0028 & 0.0157 & 0.0008 & 0.0143 & 0.0074 & 0.0124 \\
\hline & & $\left(r_{2}, r_{3}\right)$ & 0.0066 & 0.0228 & 0.0093 & 0.0160 & 0.0054 & 0.0134 & 0.0047 & 0.0108 \\
\hline \multirow{24}{*}{$(20,40)$} & \multirow{6}{*}{$\Theta_{1}$} & $\left(r_{1}, r_{1}\right)$ & 0.0093 & 0.0151 & 0.0086 & 0.0122 & 0.0036 & 0.0106 & 0.0036 & 0.0086 \\
\hline & & $\left(r_{2}, r_{2}\right)$ & 0.0012 & 0.0149 & 0.0036 & 0.0134 & 0.0057 & 0.0101 & 0.0076 & 0.0072 \\
\hline & & $\left(r_{3}, r_{3}\right)$ & 0.0076 & 0.0140 & 0.0006 & 0.0120 & 0.0006 & 0.0104 & 0.0088 & 0.0077 \\
\hline & & $\left(r_{1}, r_{2}\right)$ & 0.0034 & 0.0147 & 0.0031 & 0.0132 & 0.0093 & 0.0102 & 0.0029 & 0.0080 \\
\hline & & $\left(r_{1}, r_{3}\right)$ & 0.0062 & 0.0143 & 0.0018 & 0.0132 & 0.0065 & 0.0103 & 0.0004 & 0.0093 \\
\hline & & $\left(r_{2}, r_{3}\right)$ & 0.0026 & 0.0156 & 0.0064 & 0.0133 & 0.0044 & 0.0104 & 0.0028 & 0.0070 \\
\hline & \multirow{6}{*}{$\Theta_{2}$} & $\left(r_{1}, r_{1}\right)$ & 0.0008 & 0.0146 & 0.0044 & 0.0121 & 0.0005 & 0.0106 & 0.0009 & 0.0071 \\
\hline & & $\left(r_{2}, r_{2}\right)$ & 0.0096 & 0.0151 & 0.0094 & 0.0126 & 0.0069 & 0.0101 & 0.0029 & 0.0075 \\
\hline & & $\left(r_{3}, r_{3}\right)$ & 0.0069 & 0.0143 & 0.0006 & 0.0124 & 0.0032 & 0.0114 & 0.0009 & 0.0088 \\
\hline & & $\left(r_{1}, r_{2}\right)$ & 0.0071 & 0.0152 & 0.0035 & 0.0132 & 0.0021 & 0.0114 & 0.0044 & 0.0090 \\
\hline & & $\left(r_{1}, r_{3}\right)$ & 0.0029 & 0.0145 & 0.0079 & 0.0126 & 0.0062 & 0.0107 & 0.0004 & 0.0088 \\
\hline & & $\left(r_{2}, r_{3}\right)$ & 0.0025 & 0.0153 & 0.0022 & 0.0134 & 0.0048 & 0.0107 & 0.0099 & 0.0083 \\
\hline & \multirow{6}{*}{$\Theta_{3}$} & $\left(r_{1}, r_{1}\right)$ & 0.0062 & 0.0154 & 0.0056 & 0.0123 & 0.0051 & 0.0105 & 0.0056 & 0.0085 \\
\hline & & $\left(r_{2}, r_{2}\right)$ & 0.0014 & 0.0155 & 0.0015 & 0.0124 & 0.0083 & 0.0114 & 0.0079 & 0.0078 \\
\hline & & $\left(r_{3}, r_{3}\right)$ & 0.0004 & 0.0149 & 0.0082 & 0.0122 & 0.0046 & 0.0106 & 0.0078 & 0.0091 \\
\hline & & $\left(r_{1}, r_{2}\right)$ & 0.0076 & 0.0142 & 0.0047 & 0.0122 & 0.0053 & 0.0102 & 0.0087 & 0.0075 \\
\hline & & $\left(r_{1}, r_{3}\right)$ & 0.0018 & 0.0145 & 0.0069 & 0.0133 & 0.0062 & 0.0112 & 0.0019 & 0.0089 \\
\hline & & $\left(r_{2}, r_{3}\right)$ & 0.0055 & 0.0158 & 0.0044 & 0.0129 & 0.0043 & 0.0106 & 0.0010 & 0.0075 \\
\hline & \multirow{6}{*}{$\Theta_{4}$} & $\left(r_{1}, r_{1}\right)$ & 0.0023 & 0.0143 & 0.0012 & 0.0128 & 0.0082 & 0.0104 & 0.0027 & 0.0080 \\
\hline & & $\left(r_{2}, r_{2}\right)$ & 0.0017 & 0.0157 & 0.0005 & 0.0122 & 0.0015 & 0.0106 & 0.0053 & 0.0088 \\
\hline & & $\left(r_{3}, r_{3}\right)$ & 0.0050 & 0.0151 & 0.0009 & 0.0133 & 0.0037 & 0.0101 & 0.0026 & 0.0092 \\
\hline & & $\left(r_{1}, r_{2}\right)$ & 0.0042 & 0.0160 & 0.0075 & 0.0129 & 0.0009 & 0.0102 & 0.0054 & 0.0072 \\
\hline & & $\left(r_{1}, r_{3}\right)$ & 0.0023 & 0.0142 & 0.0004 & 0.0125 & 0.0015 & 0.0114 & 0.0087 & 0.0096 \\
\hline & & $\left(r_{2}, r_{3}\right)$ & 0.0047 & 0.0149 & 0.0089 & 0.0128 & 0.0029 & 0.0114 & 0.0095 & 0.0092 \\
\hline
\end{tabular}


192 Estimation of Reliability of Stress-strength for a Kumaraswamy Distribution ...

Table 2. Average length and coverage probability for estimations of $R$.

\begin{tabular}{|c|c|c|c|c|c|c|c|c|}
\hline \multirow[t]{2}{*}{$(n, N)$} & \multirow[t]{2}{*}{$\Theta_{j}$} & \multirow[t]{2}{*}{ C.S } & \multicolumn{3}{|c|}{ Bayes } & \multirow[t]{2}{*}{ Boot-t } & \multirow[t]{2}{*}{ Boot-p } & \multirow[t]{2}{*}{ MLE } \\
\hline & & & Prior 1 & Prior 2 & Prior 3 & & & \\
\hline \multirow{24}{*}{$(10,40)$} & \multirow{6}{*}{$\Theta_{5}$} & $\left(r_{1}, r_{1}\right)$ & $0.4331(0.947)$ & $0.4175(0.953)$ & $0.4175(0.957)$ & $0.5744(0.936)$ & $0.4928(0.941)$ & $0.4421(0.944)$ \\
\hline & & $\left(r_{2}, r_{2}\right)$ & $0.4341(0.948)$ & $0.4183(0.952)$ & $0.4183(0.956)$ & $0.5573(0.934)$ & $0.4888(0.940)$ & $0.4592(0.945)$ \\
\hline & & $\left(r_{3}, r_{3}\right)$ & $0.4263(0.949)$ & $0.4182(0.953)$ & $0.4182(0.957)$ & $0.5779(0.938)$ & $0.4705(0.942)$ & $0.4401(0.946)$ \\
\hline & & $\left(r_{1}, r_{2}\right)$ & $0.4341(0.948)$ & $0.4143(0.950)$ & $0.4143(0.958)$ & $0.5605(0.937)$ & $0.4835(0.942)$ & $0.4555(0.945)$ \\
\hline & & $\left(r_{1}, r_{3}\right)$ & $0.4313(0.947)$ & $0.4172(0.950)$ & $0.4172(0.958)$ & $0.5559(0.935)$ & $0.4765(0.940)$ & $0.4563(0.946)$ \\
\hline & & $\left(r_{2}, r_{3}\right)$ & $0.4260(0.949)$ & $0.4119(0.953)$ & $0.4119(0.957)$ & $0.5575(0.937)$ & $0.5018(0.941)$ & $0.4574(0.943)$ \\
\hline & \multirow{6}{*}{$\Theta_{6}$} & $\left(r_{1}, r_{1}\right)$ & $0.4278(0.948)$ & $0.4178(0.953)$ & $0.4178(0.957)$ & $0.5685(0.937)$ & $0.4824(0.943)$ & $0.4417(0.945)$ \\
\hline & & $\left(r_{2}, r_{2}\right)$ & $0.4305(0.949)$ & $0.4104(0.953)$ & $0.4104(0.958)$ & $0.5642(0.938)$ & $0.4911(0.943)$ & $0.4480(0.945)$ \\
\hline & & $\left(r_{3}, r_{3}\right)$ & $0.4346(0.947)$ & $0.4130(0.952)$ & $0.4130(0.958)$ & $0.5605(0.937)$ & $0.4766(0.940)$ & $0.4452(0.944)$ \\
\hline & & $\left(r_{1}, r_{2}\right)$ & $0.4346(0.948)$ & $0.410590 .953)$ & $0.4105(0.958)$ & $0.5749(0.936)$ & $0.4941(0.942)$ & $0.4560(0.944)$ \\
\hline & & $\left(r_{1}, r_{3}\right)$ & $0.4266(0.947)$ & $0.4111(0.951)$ & $0.4111(0.9570$ & $0.5676(0.934)$ & $0.4805(0.940)$ & $0.4486(0.946)$ \\
\hline & & $\left(r_{2}, r_{3}\right)$ & $0.4347(0.948)$ & $0.419190 .952)$ & $0.4191(0.957)$ & $0.5665(0.934)$ & $0.4962(0.943)$ & $0.4582(0.945)$ \\
\hline & \multirow{6}{*}{$\Theta_{7}$} & $\left(r_{1}, r_{1}\right)$ & $0.4346(0.948)$ & $0.4176(0.951)$ & $0.4176(0.957)$ & $0.5775(0.936)$ & $\begin{array}{l}0.4976(0.941) \\
\end{array}$ & $0.4436(0.943)$ \\
\hline & & $\left(r_{2}, r_{2}\right)$ & $0.4299(0.949)$ & $0.4135(0.951)$ & $0.4135(0.958)$ & $0.5586(0.938)$ & $0.4999(0.942)$ & $0.4453(0.945)$ \\
\hline & & $\left(r_{3}, r_{3}\right)$ & $0.4330(0.948)$ & $0.4205(0.953)$ & $0.4205(0.959)$ & $0.5727(0.938)$ & $0.4880(0.943)$ & $0.4429(0.946)$ \\
\hline & & $\left(r_{1}, r_{2}\right)$ & $0.4264(0.947)$ & $0.4104(0.953)$ & $0.4104(0.957)$ & $0.5726(0.935)$ & $0.4734(0.940)$ & $0.4427(0.945)$ \\
\hline & & $\left(r_{1}, r_{3}\right)$ & $0.4292(0.948)$ & $0.41489(0.952)$ & $0.4148(0.958)$ & $0.5614(0.938)$ & $0.47929(0.941)$ & $0.4574(0.946)$ \\
\hline & & $\left(r_{2}, r_{3}\right)$ & $0.4342(0.947)$ & $0.4142(0.952)$ & $0.4142(0.958)$ & $0.5670(0.937)$ & $0.5065(0.940)$ & $0.4516(0.944)$ \\
\hline & \multirow{6}{*}{$\Theta_{8}$} & $\left(r_{1}, r_{1}\right)$ & $0.4329(0.948)$ & $0.4184(0.952)$ & $0.4184(0.957)$ & $0.5523(0.934)$ & $0.4761(0.942)$ & $0.4510(0.945)$ \\
\hline & & $\left(r_{2}, r_{2}\right)$ & $0.4346(0.948)$ & $0.4187(0.953)$ & $0.4187(0.958)$ & $0.5516(0.934)$ & $0.5030(0.943)$ & $0.4429(0.946)$ \\
\hline & & $\left(r_{3}, r_{3}\right)$ & $0.4316(0.948)$ & $0.4121(0.953)$ & $0.4121(0.957)$ & $0.5659(0.938)$ & $0.4915(0.943)$ & $0.4571(0.946)$ \\
\hline & & $\left(r_{1}, r_{2}\right)$ & $0.4254(0.949)$ & $0.4154(0.954)$ & $0.4154(0.958)$ & $0.5734(0.936)$ & $0.5098(0.941)$ & $0.4524(0.945)$ \\
\hline & & $\left(r_{1}, r_{3}\right)$ & $0.4335(0.949)$ & $0.4149(0.954)$ & $0.4149(0.957)$ & $0.5780(0.936)$ & $0.4731(0.940)$ & $0.4470(0.945)$ \\
\hline & & $\left(r_{2}, r_{3}\right)$ & $0.4343(0.948)$ & $0.4171(0.951)$ & $0.4171(0.958)$ & $0.5539(0.935)$ & $0.4877(0.941)$ & $0.4503(0.944)$ \\
\hline \multirow{24}{*}{$(20,40)$} & \multirow{6}{*}{$\Theta_{5}$} & $\left(r_{1}, r_{1}\right)$ & $0.3980(0.953)$ & $0.3758(0.957)$ & $0.3349(0.961)$ & $0.4717(0.941)$ & $0.4320(0.947)$ & $0.4161(0.950)$ \\
\hline & & $\left(r_{2}, r_{2}\right)$ & $0.3915(0.955)$ & $0.3706(0.957)$ & $0.3344(0.960)$ & $0.4752(0.941)$ & $0.4352(0.945)$ & $0.4115(0.952)$ \\
\hline & & $\left(r_{3}, r_{3}\right)$ & $0.3948(0.952)$ & $0.3723(0.957)$ & $0.3345(0.961)$ & $0.4860(0.940)$ & $0.4367(0.946)$ & $0.4037(0.949)$ \\
\hline & & $\left(r_{1}, r_{2}\right)$ & $0.3925(0.953)$ & $0.3735(0.959)$ & $0.3331(0.960)$ & $0.4706(0.943)$ & $0.4436(0.945)$ & $0.4048(0.950)$ \\
\hline & & $\left(r_{1}, r_{3}\right)$ & $0.3937(0.953)$ & $0.3782(0.957)$ & $0.3351(0.962)$ & $0.4886(0.940)$ & $0.4327(0.944)$ & $0.4177(0.948)$ \\
\hline & & $\left(r_{2}, r_{3}\right)$ & $0.3948(0.950)$ & $0.3702(0.958)$ & $0.3351(0.961)$ & $0.4846(0.942)$ & $0.4444(0.946)$ & $0.4006(0.949)$ \\
\hline & \multirow{6}{*}{$\Theta_{6}$} & $\left(r_{1}, r_{1}\right)$ & $0.3983(0.950)$ & $0.3704(0.957)$ & $0.3382(0.962)$ & $0.4798(0.942)$ & $0.4321(0.945)$ & $0.4098(0.948)$ \\
\hline & & $\left(r_{2}, r_{2}\right)$ & $0.3910(0.951)$ & $0.3717(0.956)$ & $0.3379(0.962)$ & $0.4816(0.941)$ & $0.4431(0.946)$ & $0.4034(0.949)$ \\
\hline & & $\left(r_{3}, r_{3}\right)$ & $0.4081(0.952)$ & $0.3765(0.958)$ & $0.3364(0.960)$ & $0.4747(0.942)$ & $0.4399(0.945)$ & $0.4196(0.950)$ \\
\hline & & $\left(r_{1}, r_{2}\right)$ & $0.4089(0.951)$ & $0.3773(0.958)$ & $0.3338(0.962)$ & $0.4792(0.941)$ & $0.4456(0.944)$ & $0.4143(0.949)$ \\
\hline & & $\left(r_{1}, r_{3}\right)$ & $0.3998(0.953)$ & $0.3765(0.958)$ & $0.3381(0.960)$ & $0.4893(0.940)$ & $0.4443(0.944)$ & $0.4100(0.950)$ \\
\hline & & $\left(r_{2}, r_{3}\right)$ & $0.3998(0.953)$ & $0.3745(0.956)$ & $0.3353(0.962)$ & $0.4809(0.943)$ & $0.4481(0.947)$ & $0.4094(0.949)$ \\
\hline & \multirow{6}{*}{$\Theta_{7}$} & $\left(r_{1}, r_{1}\right)$ & $0.3968(0.952)$ & $0.3755(0.958)$ & $0.3335(0.961)$ & $0.4804(0.942)$ & $0.4478(0.946)$ & $0.4012(0.948)$ \\
\hline & & $\left(r_{2}, r_{2}\right)$ & $0.4080(0.950)$ & $0.3730(0.957)$ & $0.3394(0.962)$ & $0.4746(0.943)$ & $0.4367(0.946)$ & $0.4136(0.949)$ \\
\hline & & $\left(r_{3}, r_{3}\right)$ & $0.3974(0.952)$ & $0.3774(0.958)$ & $0.3388(0.961)$ & $0.4798(0.942)$ & $0.4440(0.947)$ & $0.4008(0.951)$ \\
\hline & & $\left(r_{1}, r_{2}\right)$ & $0.3922(0.952)$ & $0.3719(0.959)$ & $0.3355(0.962)$ & $0.4825(0.943)$ & $0.4340(0.947)$ & $0.4014(0.949)$ \\
\hline & & $\left(r_{1}, r_{3}\right)$ & $0.4056(0.953)$ & $0.3769(0.957)$ & $0.3362(0.961)$ & $0.4836(0.942)$ & $0.4306(0.945)$ & $0.4104(0.951)$ \\
\hline & & $\left(r_{2}, r_{3}\right)$ & $0.3978(0.953)$ & $0.3718(0.958)$ & $0.3359(0.962)$ & $0.4779(0.943)$ & $0.4449(0.947)$ & $0.4019(0.951)$ \\
\hline & \multirow{6}{*}{$\Theta_{8}$} & $\left(r_{1}, r_{1}\right)$ & $0.3948(0.951)$ & $0.3737(0.956)$ & $0.3321(0.963)$ & $0.4773(0.940)$ & $0.4400(0.946)$ & $0.4164(0.948)$ \\
\hline & & $\left(r_{2}, r_{2}\right)$ & $0.3981(0.950)$ & $0.3763(0.957)$ & $0.3330(0.960)$ & $0.4898(0.941)$ & $0.4396(0.947)$ & $0.4164(0.949)$ \\
\hline & & $\left(r_{3}, r_{3}\right)$ & $0.3919(0.952)$ & $0.3778(0.956)$ & $0.3347(0.961)$ & $0.4708(0.943)$ & $0.4481(0.946)$ & $0.4144(0.950)$ \\
\hline & & $\left(r_{1}, r_{2}\right)$ & $0.3926(0.952)$ & $0.3708(0.958)$ & $0.3323(0.962)$ & $0.4877(0.942)$ & $0.4422(0.945)$ & $0.4030(0.949)$ \\
\hline & & $\left(r_{1}, r_{3}\right)$ & $0.4088(0.953)$ & $0.3793(0.957)$ & $0.3384(0.960)$ & $0.4883(0.942)$ & $0.4424(0.947)$ & $0.4132(0.948)$ \\
\hline & & $\left(r_{2}, r_{3}\right)$ & $0.4091(0.951)$ & $0.3778(0.957)$ & $0.3319(0.963)$ & $0.4859(0.942)$ & $0.4472(0.946)$ & $0.4104(0.948)$ \\
\hline
\end{tabular}




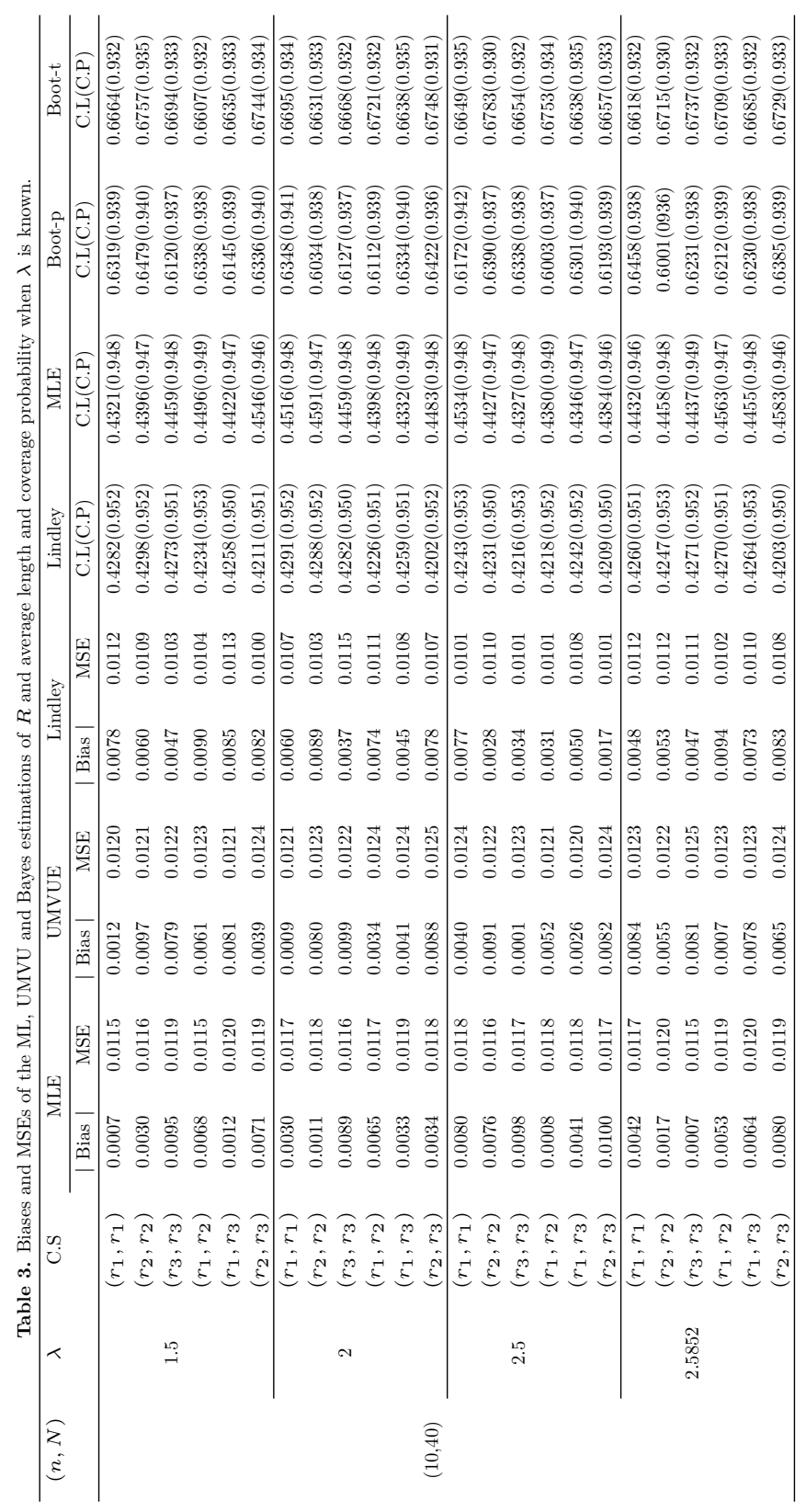




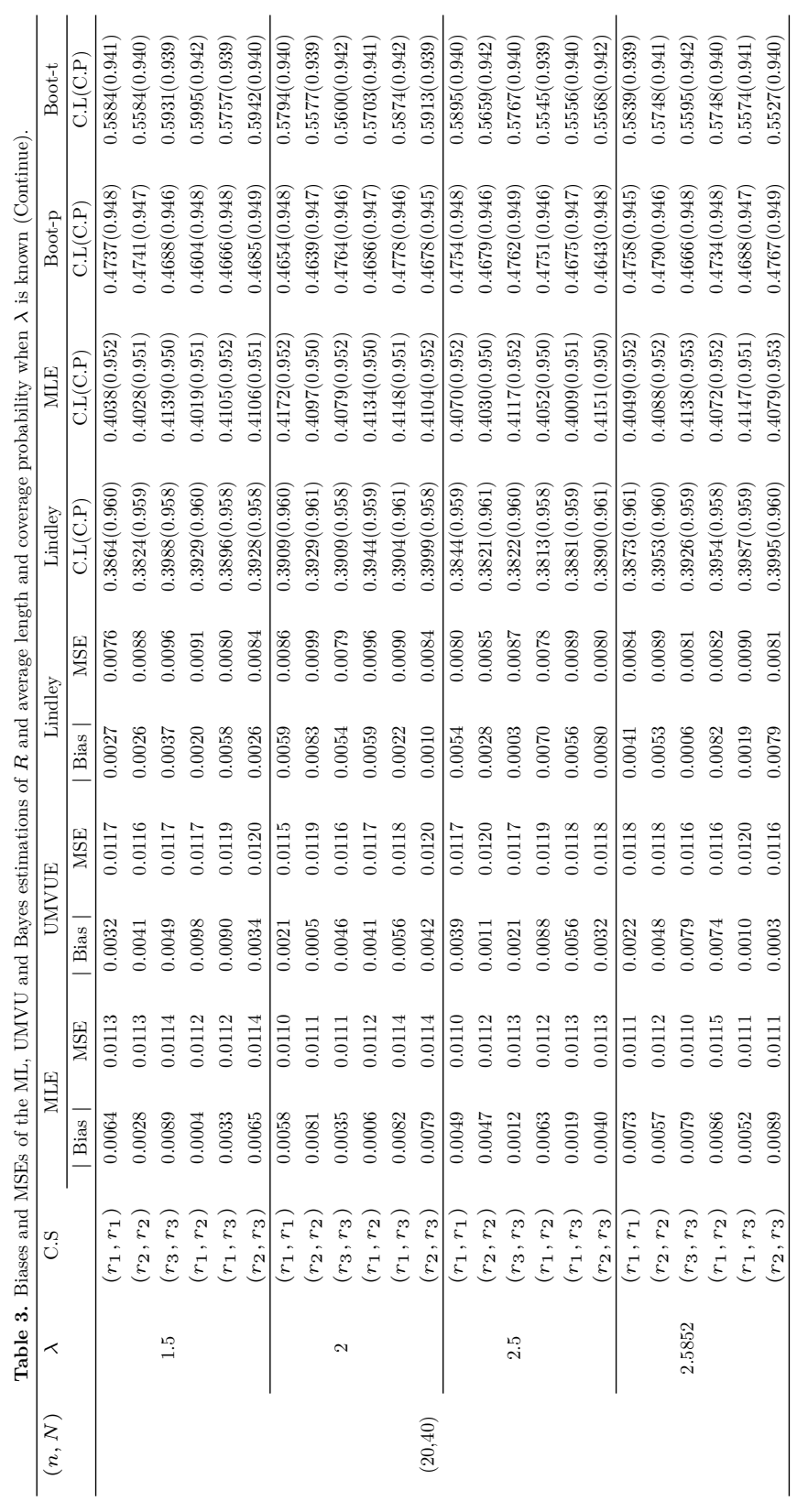




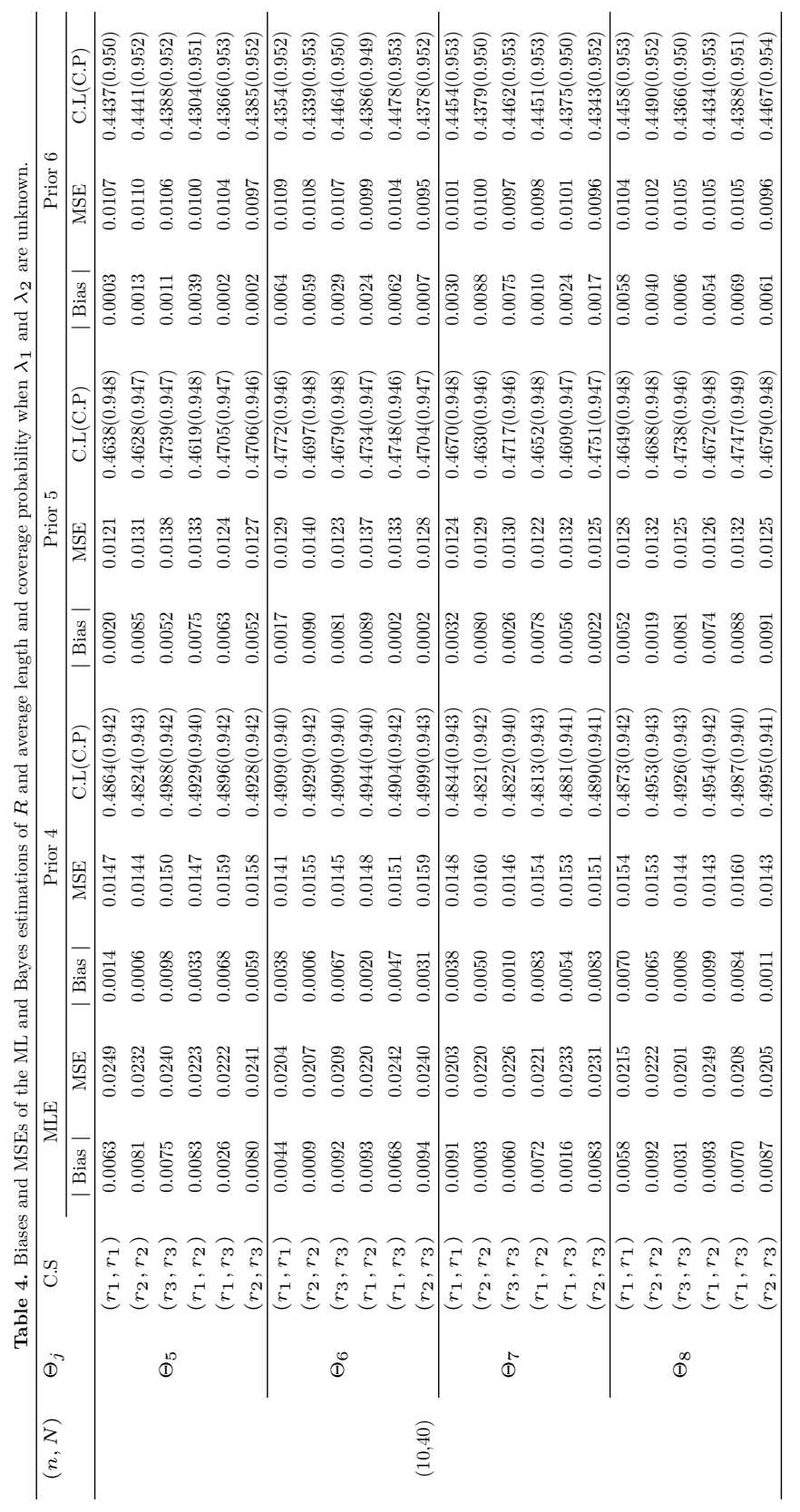




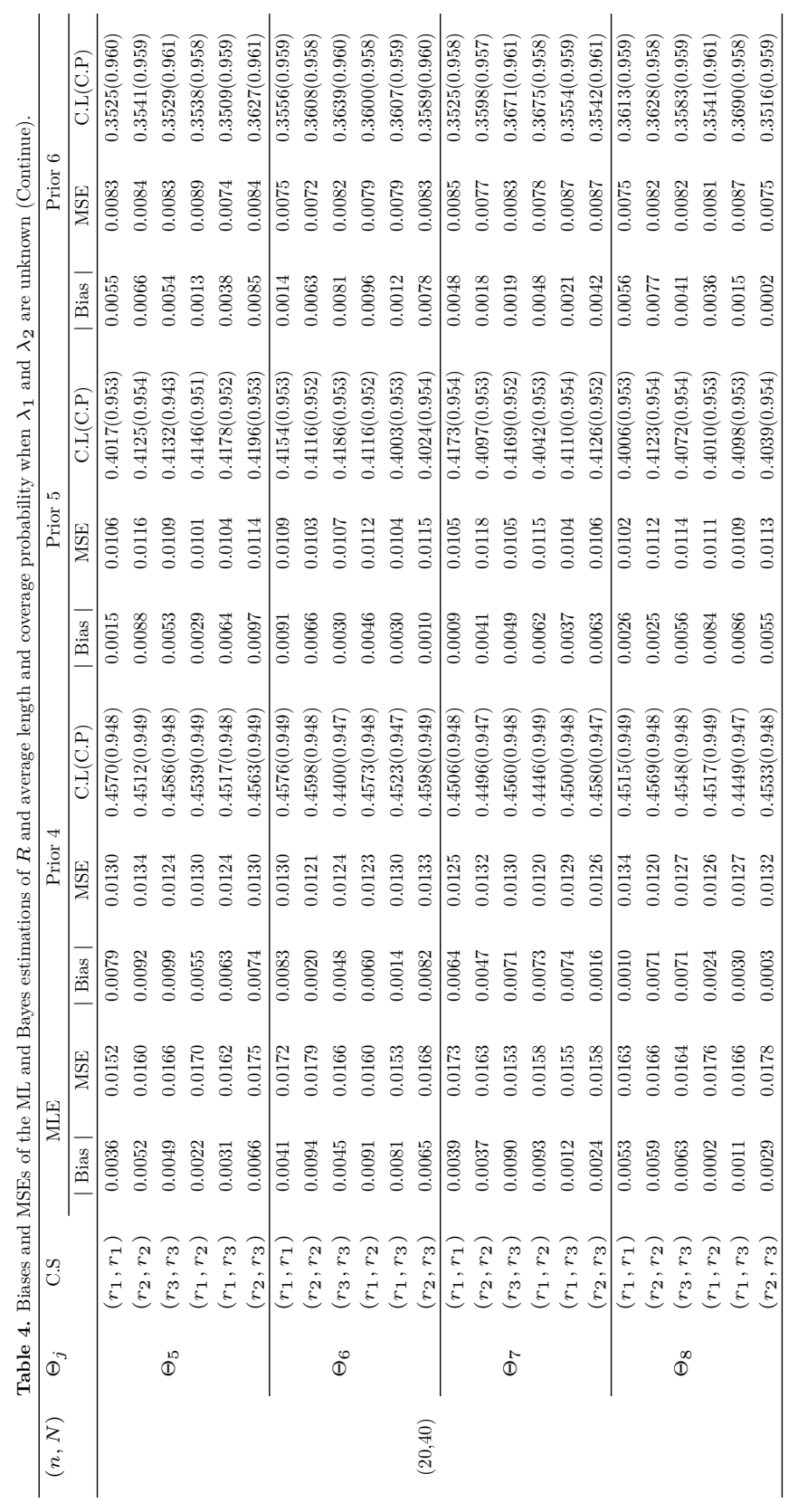


Table 5. Real data set 1

\begin{tabular}{llllllllll}
\hline \multicolumn{10}{c}{ Data set 1 } \\
\hline 1.901 & 2.132 & 2.203 & 2.228 & 2.257 & 2.350 & 2.361 & 2.396 & 2.397 & 2.445 \\
2.454 & 2.474 & 2.518 & 2.522 & 2.525 & 2.532 & 2.575 & 2.614 & 2.616 & 2.618 \\
2.626 & 2.659 & 2.675 & 2.738 & 2.740 & 2.856 & 2.917 & 2.928 & 2.937 & 2.937 \\
2.977 & 2.996 & 3.030 & 3.125 & 3.139 & 3.145 & 3.220 & 3.223 & 3.235 & 3.243 \\
3.264 & 3.272 & 3.294 & 3.332 & 3.346 & 3.377 & 3.408 & 3.435 & 3.493 & 3.501 \\
3.537 & 3.554 & 3.562 & 3.628 & 3.852 & 3.871 & 3.886 & 3.971 & 4.024 & 4.027 \\
4.225 & 4.395 & 5.020 & & & & & & & \\
\hline & & & & Transformed data & & & \\
\hline 0.128 & 0.202 & 0.225 & 0.233 & 0.242 & 0.272 & 0.276 & 0.287 & 0.287 & 0.302 \\
0.305 & 0.312 & 0.326 & 0.327 & 0.328 & 0.330 & 0.344 & 0.357 & 0.357 & 0.358 \\
0.360 & 0.371 & 0.376 & 0.396 & 0.397 & 0.434 & 0.454 & 0.457 & 0.460 & 0.460 \\
0.473 & 0.479 & 0.490 & 0.521 & 0.525 & 0.527 & 0.551 & 0.552 & 0.556 & 0.558 \\
0.565 & 0.568 & 0.575 & 0.587 & 0.591 & 0.601 & 0.611 & 0.620 & 0.638 & 0.641 \\
0.653 & 0.658 & 0.661 & 0.682 & 0.754 & 0.760 & 0.764 & 0.792 & 0.809 & 0.810 \\
0.873 & 0.928 & 1.128 & & & & & & & \\
\hline
\end{tabular}

sets. Kundu and Gupta (2006) could fit two-parameter Weibull distributions with a common shape parameter to both data sets after subtracting 0.75 from all points of the data sets. We analyze the data after applying the transformation $y=(x-a) /\left(x_{\max }-x_{\min }\right)$ on both data sets, where $a=1.5$ and 1 are selected for the first and second data set, respectively. After removing the extreme values that are greater than one (extreme values $>1$ ), the estimated parameters, for the first data set, are $\hat{\alpha}=3.5653, \hat{\lambda}=2.4854$ and for the second data set are $\hat{\beta}=2.0019, \hat{\lambda}=2.7233$. To compare different fitted distributions, we utilize the well-known information-based criteria, that are Akaike Information Criterion (AIC), Schawarz Bayesian Information Criterion (BIC) and Hannan-Quinn Criterion (HQC), whose values are given in Table 7. As shown in Table 7, the Kumaraswamy distribution is preferred over the other two distributions. For the Kumaraswamy distribution, the Kolmogorov-Smirnov distances are 0.0900 and 0.0536 and the associated $p$-values are 0.6966 and 0.9914 ( $p$-values $>0.2$ ), in the first and second data sets, respectively. It shows that the Kumaraswamy distribution fits adequately to the transformed data sets. Also, for both data sets, the PP-plots are given in Figure 2.

Because the two second shape parameters are not very different, we assume that the two parameters are approximately equal. Based on the com- 
198 Estimation of Reliability of Stress-strength for a Kumaraswamy Distribution ...

Table 6. Real data set 2

\begin{tabular}{llllllllll}
\hline \multicolumn{10}{c}{ Data set 2} \\
\hline 1.312 & 1.314 & 1.479 & 1.552 & 1.700 & 1.803 & 1.861 & 1.865 & 1.944 & 1.958 \\
1.966 & 1.997 & 2.006 & 2.021 & 2.027 & 2.055 & 2.063 & 2.098 & 2.140 & 2.179 \\
2.224 & 2.240 & 2.253 & 2.270 & 2.272 & 2.274 & 2.301 & 2.301 & 2.359 & 2.382 \\
2.382 & 2.426 & 2.434 & 2.435 & 2.478 & 2.490 & 2.511 & 2.514 & 2.535 & 2.554 \\
2.566 & 2.570 & 2.586 & 2.629 & 2.633 & 2.642 & 2.648 & 2.684 & 2.697 & 2.726 \\
2.770 & 2.773 & 2.800 & 2.809 & 2.818 & 2.821 & 2.848 & 2.880 & 2.954 & 3.012 \\
3.067 & 3.084 & 3.090 & 3.096 & 3.128 & 3.233 & 3.433 & 3.585 & 3.585 & \\
\hline \multicolumn{8}{c}{ Transformed data } \\
0.137 & 0.138 & 0.210 & 0.242 & 0.307 & 0.353 & 0.378 & 0.380 & 0.415 & 0.421 \\
0.424 & 0.438 & 0.442 & 0.449 & 0.451 & 0.464 & 0.467 & 0.483 & 0.501 & 0.518 \\
0.538 & 0.545 & 0.551 & 0.558 & 0.559 & 0.560 & 0.572 & 0.572 & 0.597 & 0.608 \\
0.608 & 0.627 & 0.630 & 0.631 & 0.650 & 0.655 & 0.664 & 0.666 & 0.675 & 0.683 \\
0.688 & 0.690 & 0.697 & 0.716 & 0.718 & 0.722 & 0.725 & 0.740 & 0.746 & 0.759 \\
0.778 & 0.780 & 0.791 & 0.795 & 0.799 & 0.801 & 0.813 & 0.827 & 0.859 & 0.885 \\
0.909 & 0.916 & 0.919 & 0.922 & 0.936 & 0.982 & 1.070 & 1.137 & 1.137 & \\
\hline
\end{tabular}
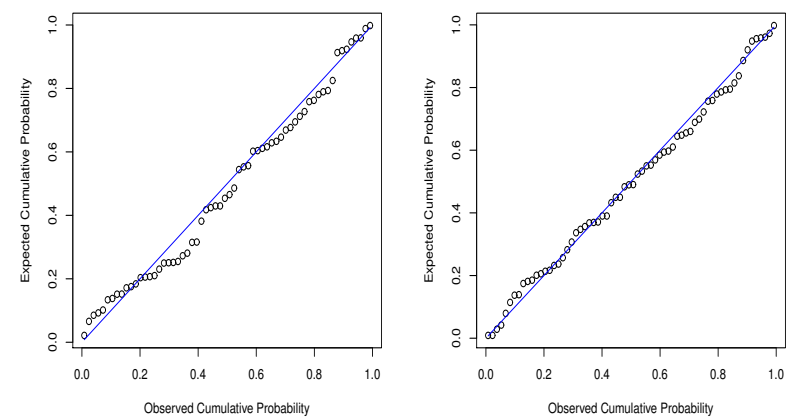

Figure 2. The PP-plots for the first (left) and second (right) data.

Table 7. The values of AIC, BIC and HQC for different fitted distributions.

\begin{tabular}{ccccccc}
\hline Distribution & \multicolumn{3}{c}{ Data set 1 } & \multicolumn{3}{c}{ Data set 2 } \\
\cline { 2 - 7 } & AIC & BIC & HQC & AIC & BIC & HQC \\
\hline generalized Rayleigh & 115.9340 & 120.2202 & 128.5065 & 105.7568 & 110.2251 & 118.6933 \\
Weibull & 124.3049 & 128.5912 & 136.8774 & 101.5606 & 106.0288 & 114.4970 \\
Kumaraswamy & 75.9262 & 80.1805 & 88.4348 & 81.0754 & 76.6961 & 68.3168 \\
\hline
\end{tabular}


plete data set, the proposed iterative procedure is used to compute the MLE. In Figure 3, the profile log-likelihood function of $\lambda$ is depicted. Because this function is unimodal, it has an unique maximum. Consequently, we begin the iterative procedure at the maximum with the initial values of $\lambda=2.5800$. By this initial value, MLEs of $\lambda, \alpha$ and $\beta$ are $(2.5852,3.7597,1.9016)$ and the ML estimation of $R$ is 0.6640 . Also, the associated $95 \%$ confidence interval is $(0.5852,0.7429)$. Regarding the improper priors, the Bayes estimation of $R$ is 0.6632 and the associated $95 \%$ credible interval is $(0.5866,0.7381)$.

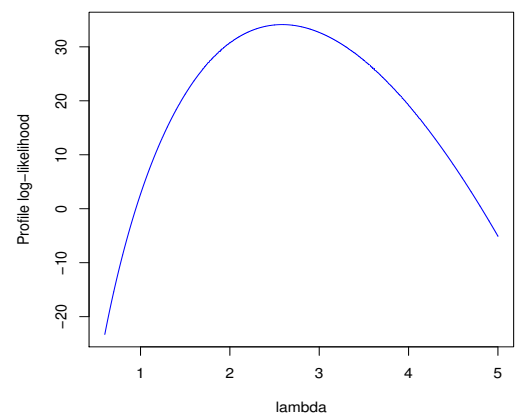

Figure 3. The profile log-likelihood function of $\lambda$.

As a final illustrative experiment, two different progressively censored samples were produced. Scheme 1: $R=[10,0,10,0,10,0,10,0,12,0], S=$ $[10,0,10,0,10,0,10,0,12,4]$ and Scheme 2: $R=[11,11,13,11,11], S=$ $[12,12,13,12,12]$. The progressive censored schemes and the related progressive censored samples are given in Table 8. Using the Scheme 1, the MLE (by means of the profile log-likelihood function) and Bayes estimate are 0.5887 and 0.6993 , respectively. The corresponding $95 \%$ asymptotic confidence interval is $(0.3768,0.8006)$ and the corresponding $95 \%$ HPD credible interval is $(0.4991,0.8542)$. Using the Scheme 2 , the ML and Bayes estimations are 0.5656 and 0.5803 . Moreover the associated $95 \%$ asymptotic confidence interval is $(0.2632,0.8679)$ and the associated $95 \%$ HPD credible interval is $(0.2920,0.8378)$. Obviously, the resulted estimations employing the Scheme 1 , are more correlated with the estimates in complete sample case compared to the Scheme 2.

Additionally, we evaluate the case of different second shape parameters. The ML estimation of $R$ is 0.6752 considering the complete data set, and with using the improper priors, the Bayes estimation of $R$ is 0.5682 and 
Table 8. The progressive censored schemes and the related progressive censored samples.

\begin{tabular}{|c|c|c|c|c|c|c|c|c|}
\hline \multicolumn{9}{|c|}{ Scheme 1: $R=[10,0,10,0,10,0,10,0,12,0], S=[10,0,10,0,10,0,10,0,12,4]$} \\
\hline \multirow[t]{2}{*}{ Censored samples } & X 0.128 & $0.202 \quad 0.225$ & $0.233 \quad 0.242$ & 0.272 & 0.276 & 0.287 & 0.287 & 0.928 \\
\hline & $\mathrm{Y} \quad 0.137$ & $0.138 \quad 0.210$ & $0.242 \quad 0.307$ & 0.353 & 0.378 & 0.380 & 0.415 & 0.982 \\
\hline \multicolumn{9}{|c|}{ Scheme $2: R=[11,11,13,11,11], S=[12,12,13,12,12]$} \\
\hline \multirow[t]{2}{*}{ Censored samples } & X 0.128 & $0.202 \quad 0.225$ & $0.233 \quad 0.928$ & & & & & \\
\hline & Y $\quad 0.137$ & $0.138 \quad 0.210$ & $0.242 \quad 0.982$ & & & & & \\
\hline
\end{tabular}

Table 9. The progressive censored schemes, the related progressive censored samples and different parameter estimations.

\begin{tabular}{|c|c|c|c|c|c|c|c|c|c|c|c|}
\hline \multirow{3}{*}{ Censored samples } & \multicolumn{11}{|c|}{ Scheme 1: $R=[10,0,10,0,10,0,10,0,12,0], S=[10,0,10,0,10,0,10,0,12,4]$} \\
\hline & $\mathrm{X}$ & 0.225 & 0.272 & 0.276 & 0.287 & 0.457 & 0.460 & 0.638 & 0.641 & 0.809 & 0.810 \\
\hline & $\mathrm{Y}$ & 0.210 & 0.380 & 0.442 & 0.467 & 0.538 & 0.551 & 0.572 & 0.688 & 0.716 & 0.919 \\
\hline & & \multicolumn{5}{|c|}{ MLE } & \multicolumn{5}{|c|}{ Bayes } \\
\hline Same unknown $\lambda$ & & \multicolumn{2}{|c|}{$\widehat{R}=0.5619$} & & \multicolumn{2}{|c|}{ C.I $=(0.3463,0.7774)$} & \multicolumn{2}{|c|}{$\widehat{R}=0.5700$} & & \multicolumn{2}{|c|}{ C.I $=(0.3471,0.7420)$} \\
\hline Different unknown $\lambda$ & & \multicolumn{2}{|c|}{$\widehat{R}=0.5852$} & & \multicolumn{2}{|r|}{ - } & \multicolumn{2}{|c|}{$\widehat{R}=0.5358$} & & \multicolumn{2}{|c|}{ C.I $=(0.3309,0.6844)$} \\
\hline \multicolumn{12}{|c|}{ Scheme 2: $R=[11,11,13,11,11], S=[12,12,13,12,12]$} \\
\hline \multirow[t]{2}{*}{ Censored samples } & $\mathrm{X}$ & 0.344 & 0.396 & 0.551 & 0.641 & 0.873 & & & & & \\
\hline & $\mathrm{Y}$ & 0.138 & 0.597 & 0.630 & 0.778 & 0.813 & & & & & \\
\hline & & \multicolumn{5}{|c|}{ MLE } & \multicolumn{5}{|c|}{ Bayes } \\
\hline Same unknown $\lambda$ & \multirow{2}{*}{\multicolumn{3}{|c|}{$\begin{aligned} R & =0.5331 \\
\widehat{R} & =0.5054\end{aligned}$}} & & \multicolumn{2}{|c|}{ C.I $=(0.2247,0.8416)$} & \multirow{2}{*}{\multicolumn{2}{|c|}{$\begin{array}{l}\widehat{R}=0.5595 \\
\widehat{R}=0.6015\end{array}$}} & & \multicolumn{2}{|c|}{ C.I $=(0.2491,0.8309)$} \\
\hline Different unknown $\lambda$ & & & & & & - & & & & C.I $=($ & $0,0.8595)$ \\
\hline
\end{tabular}

the associated $95 \%$ credible interval is $(0.4831,0.6522)$. Moreover, the ML and Bayes estimations of $R$, using the Scheme 1, are respectively 0.5684 and 0.5358 and the associated $95 \%$ HPD credible interval is $(0.3309,0.6844)$. In the same way, the ML and Bayes estimations of $R$, using the Scheme 2, are respectively 0.5342 and 0.6052 and the associated $95 \%$ HPD credible interval is $(0.2829,0.8625)$.

Because the test units are randomly removed at each failure time in the progressively Type-II censoring scheme, we implement the same progressively censoring schemes with different censoring samples. The new generated progressive censored samples and the new results are given in Table 9. Table 9 and the other results in this section, shows that, based on the Scheme 2, point and interval estimates perform worse than the point and interval estimates based on Scheme 1. In fact, we observe that the resulted estimations employing the Scheme 1, are more correlated with the estimates in complete sample case compared to the Scheme 2. Also, the different interval lengths, based on Scheme 2 is greater than the ones, based on Scheme 1. Therefore, with increasing the censoring data, we miss some useful information. 


\section{Conclusions}

In the current study, we proposed some approaches for estimation of the stress-strength parameter for KuD assuming progressive Type-II censoring scheme. For performance comparisons of the proposed estimators, Monte Carlo simulations and real data analysis have been provided. Three cases have been considered, first the common second shape parameter is assumed unknown, second it is assumed known, and finally the general case is studied where the second shape parameters are different and unknown. In case of the unknown common second shape parameter, different methods for estimating $R=P(X<Y)$ have been presented. We observed that the MLE of $R$ can not be obtained in a closed form, so an algorithm is applied to compute it iteratively. Moreover, we achieved the asymptotic confidence interval using Fisher information matrix. It was demonstrated that in case of rather small sample sizes, the asymptotic confidence intervals perform quite well. Additionally, two proposed bootstrap confidence intervals have performed quite satisfactory. By means of the Gibbs sampling technique, the Bayes estimation of $R$ and the corresponding credible interval have been achieved. We observed that the MLE is comparable to the Bayes estimation in the sense of the resulted biases and MSEs. Also, in case of the known second shape parameter, MLE, UMVUE, exact confidence interval of $R$ and different Bayes estimators have been computed. We observed that MLE provides the smallest biases, and MSEs and the UMVUEs are the best second estimators, respectively. Also, the Lindley approximation behaves quite differently from the other estimation strategies. Assuming all four shape parameters are unknown and different, the ML and Bayes estimations of $R$ have been also studied. In this case numerical computation is utilized to infer about $R$, as $R$ cannot be obtained in a closed form. The Bayes estimation of $R$ and the associated credible interval were obtained utilizing the Gibbs sampling method. The simulation results show that the MLE lead to a comparable performance with the Bayes estimation in the sense of biases and MSEs.

This study has the potential to be employed in the context of reliability theory and censored data analysis. For future work, the proposed progressive censored scheme can be extended to the progressive hybrid $\mathrm{KuD}$ and adaptive progressive hybrid censored $\mathrm{KuD}$. 


\section{References}

Ahmad, K.E., Fakhry, M.E. and Jaheen, Z.F. (1997). Empirical Bayes Estimation of $P(Y<$ $X)$ and Characterization of Burr-Type X Model. Journal of Statistical Planning and Inference, 64, 297-308.

Ahmadi, K. and Ghafouri, S. (2019). Reliability Estimation in a Multicomponent Stressstrength Model under Generalized Half-normal Distribution based on Progressive Type-II Censoring. Journal of Statistical Computation and Simulation, 89, 2505-2548.

Asgharzadeh, A., Valiollahi, R. and Raqab, M.Z. (2011). Stress-strength Reliability of Weibull Distribution based on Progressively Censored Samples. SORT, 35, 103-124.

Badar, M.G. and Priest, A.M. (1982). Statistical Aspects of Fiber and Bundle Strength in Hybrid Composites, in: Progress in Science and Engineering Composites, Hayashi, T., Kawata, K. and Umekawa, S. Eds., Tokyo. 1129-1136, ICCM-IV.

Balakrishnan, N. and Aggarwala, R. (2000). Progressive Censoring: Theory, Methods and Applications. Birkhauser, Boston.

Baklizi, A. (2007). Inference about the Mean Difference of Two Non-normal Populations based on Independent Samples: a Comparative Study. Journal of Statistical Computation and Simulation, 77, 613-623.

Birnbaum, Z.W. (1956). On a Use of Mann-Whitney Statistics. Proceedings of the Third Berkley Symposium in Mathematics, Statistics and Probability, 1, 13-17.

Cao, J.H. and Cheng, K. (2006). An Introduction to the Reliability Mathematics. Beijing: Higher Education Press.

Chen, M.H. and Shao, Q.M. (1999). Monte Carlo Estimation of Bayesian Credible and HPD Intervals. Journal of Computational and Graphical Statistics, 8, 69-92.

Efron, B. (1982). The Jackknife, the Bootstrap and Other Re-sampling Plans. Philadelphia, PA: SIAM, CBMSNSF Regional Conference Series in Applied Mathematics, 34.

Gradshteyn, I.S. and Ryzhik, I. M. (1994). Table of Integrals, Series, and Products. 5th ed., Academic Press, Boston, MA.

Hall, P. (1988). Theoretical Comparison of Bootstrap Confidence Intervals. Annals of Statistics, 16, 927-953.

Johnson, N.L., Kotz, S. and Balakrishnan, N. (1994). Continuous Univariate Distributions. 2nd ed., Wiley, NewYork.

Jones, M. C. (2009). Kumaraswamy's Distribution: A beta-type Distribution with Some Tractability Advantages. Statistical Methodology, 6, 70-81. 
Kotz, S., Lumelskii, Y. and Pensky, M. (2003). The Stress-strength Model and Its Generalization: Theory and Applications. World Scientific, Singapore.

Kundu, D. and Gupta, R.D. (2006). Estimation of $P[Y<X]$ for Weibull Distributions. IEEE Transactions on Reliability, 55, 270-280.

Lemonte, A.J. (2011). Improved Point Estimation for the Kumaraswamy Distribution. Journal of Statistical Computation and Simulation, 81, 1971-1982.

Li, J. and Fine, J.P. (2010). Weighted Area under the Receiver Operating Characteristic Curve and Its Application to Gene Selection. Journal of the Royal Statistical Society: Series C (Applied Statistics), 59, 673-692.

Li, J. and Ma, S. (2011). Time-dependent ROC Analysis under Diverse Censoring Patterns. Statistics in Medicine, 30, 1266-1277.

Lindley, D.V. (1980). Approximate Bayesian Methods. Trabajos de Estadistica, 3, 281-288.

Mirjalili, S.M., Torabi, H., Nadeb, H. and Bafekri, S.F. (2016). Stress-strength Reliability of Exponential Distribution based on Type-I Progressively Hybrid Censored Samples. Journal of Statistical Research of Iran, 13, 89-105.

Mitnik, P.A. (2013). New Properties of the Kumaraswamy Distribution. Communications in Statistics - Theory and Methods, 42, 741-755.

Nadar, M., Papadopoulos, A. and Kizilaslan, F. (2013). Statistical Analysis for Kumaraswamy's Distribution based on Record Data. Statistical Papers, 54, 355-369.

Nadar, M., Kizilaslan, F. and Papadopoulos, A. (2014). Classical and Bayesian Estimation of $P(Y<X)$ for Kumaraswamy's Distribution. Journal of Statistical Computation and Simulation, 84, 1505-1529.

Nadar, M. and Kizilaslan, F. (2014). Classical and Bayesian Estimation of $P(X<Y)$ using Upper Record Values from Kumaraswamy's Distribution. Statistical Papers, 55, 751-783.

Raqab, M.Z. and Kundu, D. (2005). Comparison of Different Estimators of $P(Y<X)$ for a Scaled Burr Type X Distribution. Communications in Statistics - Simulation and Computation, 34, 465-483.

Shoaee, S. and Khorram, E. (2015). Stress-strength Reliability of a two-parameter BathtubShaped Lifetime Distribution based on Progressively Censored Samples. Communications in Statistics - Theory and Methods, 44, 5306-5328.

Surles, J.G. and Padgett, W.J. (1998). Inference for $P(Y<X)$ in the Burr Type X Model. Journal of Applied Statistical Sciences, 7, 225-238.

Surles, J.G. and Padgett, W.J. (2001). Inference for Reliability and Stress-strength for a Scaled Burr-type X Distribution. Lifetime Data Analysis, 7, 187-200. 
Wang, B.X., Wang, X.K. and Yu, K. (2016). Inference on the Kumaraswamy Distribution. Communications in Statistics - Theory and Methods. Accepted. DOI: 10.1080/03610926.2015.1032425.

\section{Appendix A}

The elements of $J(\theta)$ are as follows:

$$
\begin{array}{rlr}
J_{11}= & \frac{n}{\alpha^{2}}, J_{22}=\frac{m}{\beta^{2}}, J_{12}=J_{21}=0, & \\
J_{14}=J_{41}=0, J_{23}=J_{32}=0, J_{34}=J_{43}=0, & \\
J_{13}=J_{31}= & \frac{\alpha}{\lambda_{1}} \sum_{i=1}^{n}\left(R_{i}+1\right) C_{i-1} \sum_{k=1}^{i} a_{i, k} B\left(2, \alpha \eta_{k}-1\right)\left[\psi(2)-\psi\left(\alpha \eta_{k}+1\right)\right], \\
J_{24}=J_{42}= & \text { for } \alpha \eta_{k}>1 \\
\lambda_{2} \sum_{j=1}^{m}\left(S_{j}+1\right) C_{j-1}^{\prime} \sum_{k=1}^{j} a_{j, k}^{\prime} B\left(2, \beta \eta_{k}^{\prime}-1\right)\left[\psi(2)-\psi\left(\beta \eta_{k}^{\prime}+1\right)\right], & \text { for } \beta \eta_{k}^{\prime}>1 \\
J_{33}=\frac{n}{\lambda_{1}^{2}}+\frac{\alpha}{\lambda_{1}^{2}} \sum_{i=1}^{n}\left(\alpha\left(R_{i}+1\right)-1\right) C_{i-1} \sum_{k=1}^{i} a_{i, k} B\left(2, \alpha \eta_{k}-2\right) & \\
& \times\left\{\psi^{\prime}(2)-\psi^{\prime}\left(\alpha \eta_{k}\right)+\left[\psi(2)-\psi\left(\alpha \eta_{k}\right)\right]^{2}\right\} & \\
J_{44}=\frac{m}{\lambda_{2}^{2}}+\frac{\beta}{\lambda_{2}^{2}} \sum_{j=1}^{m}\left(\beta\left(S_{j}+1\right)-1\right) C_{j-1}^{\prime} \sum_{k=1}^{j} a_{j, k}^{\prime} B\left(2, \beta \eta_{k}^{\prime}-2\right) & \\
\quad \times\left\{\psi^{\prime}(2)-\psi^{\prime}\left(\beta \eta_{k}^{\prime}\right)+\left[\psi(2)-\psi\left(\beta \eta_{k}^{\prime}\right)\right]^{2}\right\} & \text { for } \beta \eta_{k}>2,
\end{array}
$$

Theorem 5. As $n \rightarrow \infty$ and $m \rightarrow \infty$ then

$\left[\sqrt{n}(\widehat{\alpha}-\alpha) \sqrt{m}(\widehat{\beta}-\beta) \sqrt{n}\left(\widehat{\lambda}_{1}-\lambda_{1}\right) \sqrt{m}\left(\widehat{\lambda}_{2}-\lambda_{2}\right)\right]^{T} \stackrel{D}{\longrightarrow} N_{4}\left(0, \mathbf{A}^{-\mathbf{1}}\left(\alpha, \beta, \lambda_{1}, \lambda_{2}\right)\right)$, where $\mathbf{A}\left(\alpha, \beta, \lambda_{1}, \lambda_{2}\right)$ and $\mathbf{A}^{-\mathbf{1}}\left(\alpha, \beta, \lambda_{1}, \lambda_{2}\right)$ are symmetric matrices and

$$
\mathbf{A}\left(\alpha, \beta, \lambda_{1}, \lambda_{2}\right)=\left[a_{i j}\right], \quad \mathbf{A}^{-1}\left(\alpha, \beta, \lambda_{1}, \lambda_{2}\right)=\frac{\left[b_{i j}\right]}{\left|\mathbf{A}\left(\alpha, \beta, \lambda_{1}, \lambda_{2}\right)\right|},
$$


in which

$$
\begin{aligned}
& a_{11}=\lim _{n, m \rightarrow \infty} \frac{J_{11}}{n}, \quad a_{13}=\lim _{n, m \rightarrow \infty} \frac{J_{13}}{n}, \quad a_{22}=\lim _{n, m \rightarrow \infty} \frac{J_{22}}{m}, \quad a_{12}=0, \quad a_{14}=0, \\
& \begin{aligned}
a_{24}=\lim _{n, m \rightarrow \infty} \frac{J_{24}}{m}, \quad a_{33}=\lim _{n, m \rightarrow \infty} \frac{J_{33}}{n}, \quad a_{44}=\lim _{n, m \rightarrow \infty} \frac{J_{44}}{m}, \quad a_{23}=0, \quad a_{34}=0, \\
\text { and }\left|\mathbf{A}\left(\alpha, \beta, \lambda_{\mathbf{1}}, \lambda_{\mathbf{2}}\right)\right|=a_{11} a_{22} a_{33} a_{44}+a_{13}^{2} a_{24}^{2}-a_{11} a_{24}^{2} a_{33}-a_{13}^{2} a_{22} a_{44}, \\
b_{11}=a_{22} a_{33} a_{44}-a_{24}^{2} a_{33}, \quad b_{12}=0, \quad b_{13}=a_{13} a_{24}^{2}-a_{13} a_{22} a_{44}, \quad b_{14}=0, \\
b_{22}=a_{11} a_{33} a_{44}-a_{13}^{2} a_{44}, \quad b_{23}=0, \quad b_{24}=a_{13}^{2} a_{24}-a_{11} a_{24} a_{33}, \\
b_{33}=a_{11} a_{22} a_{44}-a_{11} a_{24}^{2}, \quad b_{34}=0, \\
b_{44}=a_{11} a_{22} a_{33}-a_{13}^{2} a_{22} .
\end{aligned}
\end{aligned}
$$

Proof. Following the asymptotic normality of the MLE, the theorem is proved.

\section{Appendix B}

Proof of Theorem 3: Observe that $X_{1: n: N}^{*}$ and $Y_{1: m: M}^{*}$ follow an exponential distribution with mean $(N \alpha)^{-1}$ and $(M \beta)^{-1}$, respectively. Then,

$$
\phi\left(X_{1}^{*}, Y_{1}^{*}\right)=\left\{\begin{aligned}
1 & \text { if } M Y_{1: m: M}^{*}>N X_{1: n: N}^{*}, \\
0 & \text { if } M Y_{1: m: M}^{*}<N X_{1: n: N}^{*},
\end{aligned}\right.
$$

is an unbiased estimation of $R$. So,

$$
\tilde{R}=E\left[\phi\left(X_{1}^{*}, Y_{1}^{*}\right) \mid U=u, V=v\right]=\iint_{\mathcal{A}} f_{X_{1}^{*} \mid U=u}(x) f_{Y_{1}^{*} \mid V=v}(y) d x d y,
$$

where $\mathcal{A}=\{(x, y): 0<x<u / N, 0<y<v / M, N x<M y\}, f_{X_{1}^{*} \mid U=u}(x)$ and $f_{Y_{1}^{*} \mid V=v}(y)$ are the same as defined in Lemma 1. For $v<u$ 


$$
\begin{gathered}
\tilde{R}=\frac{N(n-1)}{u^{n-1}} \frac{M(m-1)}{v^{m-1}} \int_{0}^{v / M} \int_{0}^{M y / N}(u-N x)^{n-2}(v-M y)^{m-2} d x d y \\
=1-\frac{M(m-1)}{u^{n-1} v^{m-1}} \int_{0}^{v / M}(v-M y)^{m-2}(u-M y)^{n-1} d y \quad\left\{P u t: \frac{M y}{v}=t\right\} \\
=1-(m-1) \int_{0}^{1}(1-t)^{m-2}\left(1-\frac{v t}{u}\right)^{n-1} d t \\
=1-(m-1) \int_{0}^{1}(1-t)^{m-2} \sum_{k=0}^{n-1}(-1)^{k}\left(\begin{array}{c}
n-1 \\
k
\end{array}\right)\left(\frac{v t}{u}\right)^{k} d t \\
=1-\sum_{k=0}^{n-1}(-1)^{k}\left(\frac{v}{u}\right)^{k} \frac{\left(\begin{array}{c}
n-1 \\
k
\end{array}\right)}{\left(\begin{array}{c}
m+k-1 \\
k
\end{array}\right)} .
\end{gathered}
$$

Similarly for $v>u$

$$
\begin{aligned}
\tilde{R} & =\frac{N(n-1)}{u^{n-1}} \frac{M(m-1)}{v^{m-1}} \int_{0}^{u / N} \int_{N x / M}^{v / M}(u-N x)^{n-2}(v-M y)^{m-2} d y d x \\
& =\frac{N(n-1)}{u^{n-1} v^{m-1}} \int_{0}^{u / N}(u-N x)^{n-2}(v-N x)^{m-1} d x \quad\left\{P u t: \frac{N x}{u}=t\right\} \\
& =(n-1) \int_{0}^{1}(1-t)^{n-2}\left(1-\frac{u t}{v}\right)^{m-1} d t \\
& =(n-1) \int_{0}^{1}(1-t)^{n-2} \sum_{k=0}^{m-1}(-1)^{k}\left(\begin{array}{c}
m-1 \\
k
\end{array}\right)\left(\frac{u t}{v}\right)^{k} d t \\
& =\sum_{k=0}^{m-1}(-1)^{k}\left(\frac{u}{v}\right)^{k} \frac{\left(\begin{array}{c}
m-1 \\
k
\end{array}\right)}{\left(\begin{array}{c}
n+k-1 \\
k
\end{array}\right)} .
\end{aligned}
$$

\section{Appendix C}

Proof of Lemma 3: Let $\xi(\lambda)=\frac{\log \left(1-a_{i}^{\lambda}\right)}{\log \left(1-a_{1}^{\lambda}\right)}: 0<a_{1}<a_{i}<1, i=2, \ldots, n$. This function is strictly decreasing in $\lambda$ since the first derivative of $\xi(\lambda)$ is 


$$
\begin{aligned}
\frac{d \xi(\lambda)}{d \lambda} & =\frac{1}{\log ^{2}\left(1-a_{1}^{\lambda}\right)}\left[-\log \left(1-a_{1}^{\lambda}\right) \frac{a_{i}^{\lambda} \log \left(a_{i}\right)}{1-a_{i}^{\lambda}}+\log \left(1-a_{i}^{\lambda}\right) \frac{a_{1}^{\lambda} \log \left(a_{1}\right)}{1-a_{1}^{\lambda}}\right] \\
& =\frac{-1}{\log ^{2}\left(1-a_{1}^{\lambda}\right)}\left[\log \left(\frac{1}{1-a_{1}^{\lambda}}\right) \frac{a_{i}^{\lambda} \log \left(\frac{1}{a_{i}}\right)}{1-a_{i}^{\lambda}}-\log \left(\frac{1}{1-a_{i}^{\lambda}}\right) \frac{a_{1}^{\lambda} \log \left(\frac{1}{a_{1}}\right)}{1-a_{1}^{\lambda}}\right] \\
& <\frac{-1}{\log ^{2}\left(1-a_{1}^{\lambda}\right)}\left[\log \left(\frac{1}{1-a_{i}^{\lambda}}\right) \frac{a_{i}^{\lambda} \log \left(\frac{1}{a_{1}}\right)}{1-a_{i}^{\lambda}}-\log \left(\frac{1}{1-a_{i}^{\lambda}}\right) \frac{a_{1}^{\lambda} \log \left(\frac{1}{a_{1}}\right)}{1-a_{1}^{\lambda}}\right] \\
& =\frac{-1}{\log ^{2}\left(1-a_{1}^{\lambda}\right)} \log \left(\frac{1}{1-a_{i}^{\lambda}}\right) \log \left(\frac{1}{a_{1}}\right)\left[\frac{a_{i}^{\lambda}}{1-a_{i}^{\lambda}}-\frac{a_{1}^{\lambda}}{1-a_{1}^{\lambda}}\right]<0 .
\end{aligned}
$$

Moreover, after simplifying $T_{X}(\lambda)$, this expression becomes

$$
\begin{aligned}
T_{X}(\lambda) & =\frac{U_{X}}{(n-1) V_{X}}=\frac{\sum_{i=1}^{n} Z_{i}^{*}-Z_{1}^{*}}{(n-1) Z_{1}^{*}} \\
& =\frac{1}{N(n-1)} \sum_{i=1}^{n}\left(R_{i}+1\right) \frac{\log \left(1-X_{i: n: N}^{\lambda}\right)}{\log \left(1-X_{1: n: N}^{\lambda}\right)}-\frac{1}{n-1}
\end{aligned}
$$

Hence, it is easy to see that $T_{X}(\lambda)$ is a strictly decreasing function of $\lambda$.

\section{Appendix D}

Proof of Theorem 4:

(i) By using Lemma 2 and Lemma 3, we have

$$
\begin{aligned}
& 1-\gamma=\sqrt{1-\gamma} \cdot \sqrt{1-\gamma} \\
& =P\left[F_{(1-\sqrt{1-\gamma}) / 2}(2 n-2,2) \leq T_{X}(\lambda) \leq F_{(1+\sqrt{1-\gamma}) / 2}(2 n-2,2)\right] \\
& \quad \times P\left[F_{(1-\sqrt{1-\gamma}) / 2}(2 m-2,2) \leq T_{Y}(\lambda) \leq F_{(1+\sqrt{1-\gamma}) / 2}(2 m-2,2)\right]
\end{aligned}
$$




$$
\begin{aligned}
= & P\left[F_{(1-\sqrt{1-\gamma}) / 2}(2 n-2,2) \leq T_{X}(\lambda) \leq F_{(1+\sqrt{1-\gamma}) / 2}(2 n-2,2),\right. \\
& \left.F_{(1-\sqrt{1-\gamma}) / 2}(2 m-2,2) \leq T_{Y}(\lambda) \leq F_{(1+\sqrt{1-\gamma}) / 2}(2 m-2,2)\right] \\
= & P\left[T_{X}^{-1}\left(F_{(1+\sqrt{1-\gamma}) / 2}(2 n-2,2)\right) \leq \lambda \leq T_{X}^{-1}\left(F_{(1-\sqrt{1-\gamma}) / 2}(2 n-2,2)\right),\right. \\
& \left.T_{Y}^{-1}\left(F_{(1+\sqrt{1-\gamma}) / 2}(2 m-2,2)\right) \leq \lambda \leq T_{Y}^{-1}\left(F_{(1-\sqrt{1-\gamma}) / 2}(2 m-2,2)\right)\right] \\
= & P\left[\max \left\{T_{X}^{-1}\left(F_{(1+\sqrt{1-\gamma}) / 2}(2 n-2,2)\right), T_{Y}^{-1}\left(F_{(1+\sqrt{1-\gamma}) / 2}(2 m-2,2)\right)\right\} \leq \lambda\right. \\
& \left.\leq \min \left\{T_{X}^{-1}\left(F_{(1-\sqrt{1-\gamma}) / 2}(2 n-2,2)\right), T_{Y}^{-1}\left(F_{(1-\sqrt{1-\gamma}) / 2}(2 m-2,2)\right)\right\}\right] .
\end{aligned}
$$

(ii) By using Lemma 2, Lemma 3 and Lemma 4, we have

$$
\begin{aligned}
1-\gamma=\sqrt[4]{1-\gamma} \cdot \sqrt[4]{1-\gamma} \cdot \sqrt{1-\gamma} & \\
=P[ & \left.F_{(1-\sqrt[4]{1-\gamma}) / 2}(2 n-2,2) \leq T_{X}(\lambda) \leq F_{(1+\sqrt[4]{1-\gamma}) / 2}(2 n-2,2)\right] \\
& \times P\left[F_{(1-\sqrt[4]{1-\gamma}) / 2}(2 m-2,2) \leq T_{Y}(\lambda) \leq F_{(1+\sqrt[4]{1-\gamma}) / 2}(2 m-2,2)\right] \\
& \times P\left[F_{(1-\sqrt{1-\gamma}) / 2}(2 n, 2 m) \leq F \leq F_{(1+\sqrt{1-\gamma}) / 2}(2 n, 2 m)\right] \\
=P[ & F_{(1-\sqrt[4]{1-\gamma}) / 2}(2 n-2,2) \leq T_{X}(\lambda) \leq F_{(1+\sqrt[4]{1-\gamma}) / 2}(2 n-2,2), \\
& F_{(1-\sqrt[4]{1-\gamma}) / 2}(2 m-2,2) \leq T_{Y}(\lambda) \leq F_{(1+\sqrt[4]{1-\gamma}) / 2}(2 m-2,2), \\
& F_{(1-\sqrt{1-\gamma}) / 2}(2 n, 2 m) \leq \frac{R}{1-R} \cdot \frac{\left.1-\widehat{R}_{M L} \leq F_{(1+\sqrt{1-\gamma}) / 2}(2 n, 2 m)\right]}{\widehat{R}_{M L}} \\
=P[ & T_{X}^{-1}\left(F_{(1+\sqrt[4]{1-\gamma}) / 2}(2 n-2,2)\right) \leq \lambda \leq T_{X}^{-1}\left(F_{(1-\sqrt[4]{1-\gamma}) / 2}(2 n-2,2)\right), \\
& T_{Y}^{-1}\left(F_{(1+\sqrt[4]{1-\gamma}) / 2}(2 m-2,2)\right) \leq \lambda \leq T_{Y}^{-1}\left(F_{(1-\sqrt[4]{1-\gamma}) / 2}(2 m-2,2)\right), \\
& \left.F_{1-(1+\sqrt{1-\gamma}) / 2}(2 m, 2 n) \leq \frac{1-R}{R} \cdot \frac{\widehat{R}_{M L}}{1-\widehat{R}_{M L}} \leq F_{1-(1-\sqrt{1-\gamma}) / 2}(2 m, 2 n)\right]
\end{aligned}
$$




$$
\begin{gathered}
=P\left[\max \left\{T_{X}^{-1}\left(F_{(1+\sqrt[4]{1-\gamma}) / 2}(2 n-2,2)\right), T_{Y}^{-1}\left(F_{(1+\sqrt[4]{1-\gamma}) / 2}(2 m-2,2)\right)\right\} \leq \lambda\right. \\
\leq \min \left\{T_{X}^{-1}\left(F_{(1-\sqrt[4]{1-\gamma}) / 2}(2 n-2,2)\right), T_{Y}^{-1}\left(F_{(1-\sqrt[4]{1-\gamma}) / 2}(2 m-2,2)\right)\right\} \\
\left.\frac{1}{1+\frac{1-\widehat{R}_{M L}}{\widehat{R}_{M L}} F_{1-(1-\sqrt{1-\gamma}) / 2}(2 m, 2 n)} \leq R \leq \frac{1}{1+\frac{1-\widehat{R}_{M L}}{\widehat{R}_{M L}} F_{1-(1+\sqrt{1-\gamma}) / 2}(2 m, 2 n)}\right]
\end{gathered}
$$

\section{Akram Kohansal}

Department of Statistics,

Imam Khomeini International University, Qazvin, Iran.

email:kohansal@sci.ikiu.ac.ir

\section{Ramin Kazemi}

Department of Statistics,

Imam Khomeini International University, Qazvin, Iran.

email: r.kazemi@sci.ikiu.ac.ir 
